\title{
Infrared singularities in Landau gauge Yang-Mills theory
}

\author{
Reinhard Alkofer, Markus Q. Huber and Kai Schwenzer \\ Institut für Physik, Karl-Franzens Universität Graz, Universitätsplatz 5, 8010 Graz, Austria
}

\begin{abstract}
We present a more detailed picture of the infrared regime of Landau gauge Yang-Mills theory. This is done within a novel framework that allows one to take into account the influence of finite scales within an infrared power counting analysis. We find that there are two qualitatively different infrared fixed points of the full system of Dyson-Schwinger equations. The first extends the known scaling solution, where the ghost dynamics is dominant and gluon propagation is strongly suppressed. It features in addition to the strong divergences of gluonic vertex functions in the previously considered uniform scaling limit, when all external momenta tend to zero, also weaker kinematic divergences, when only some of the external momenta vanish. The second solution represents the recently proposed decoupling scenario where the gluons become massive and the ghosts remain bare. In this case we find that none of the vertex functions is enhanced, so that the infrared dynamics is entirely suppressed. Our analysis also provides a strict argument why the Landau gauge gluon dressing function cannot be infrared divergent.
\end{abstract}

\section{INTRODUCTION}

The combined effort of functional approaches and lattice gauge theory led in the past years to a comprehensive picture of the qualitative features of the infrared (IR) limit of Yang-Mills theory in Landau gauge [1-3]. This qualitative information is encoded in a set of IR power laws for the Green functions of the theory. These can incorporate important aspects of the confinement mechanism for gluons within the scenarios of Kugo-Ojima [4] and Gribov-Zwanziger [5] and serve as a basis for the inclusion of matter fields and the challenging problems of quark confinement [6] 8 and spontaneous chiral symmetry breaking [2, 9,

Previous studies of the Dyson-Schwinger equations (DSEs) [1, 2, 10, suggested that the qualitative aspects of the Yang-Mills IR fixed point structure in Landau gauge are already known [11-14. It had been shown that when the DSEs and functional renormalization group equations are combined, within the class of scaling fixed points, where no masses are dynamically generated, there exists a unique solution [15]. This conclusion was possible by the complementary constraints obtained from the two different hierarchies of equations and we will show here that, taking into account the constraints provided by the existence of the skeleton expansion, this is already implied by the DSE system alone. Yet, analog to the propagators, which depend on a single external scale, it was expected that the IR limit of a Green function is uniquely determined by a single IR scaling law. In the case of the vertices, however, the situation is more diverse. Besides the appearance of a multitude of different tensor structures, the corresponding form factors are also functions of several distinct momenta. Therefore, there are in general different combinations of momenta that can become soft and lead to IR divergences. In present studies the implicit assumption was that vertices become IR divergent if and only if all scales go to zero. This led to general results for the IR scaling of arbitrary vertices in this uniform limit [14 that were later extended to arbitrary dimension [16. Here we show that this picture - although qualitatively correct - needs to be refined in the sense that there are additional kinematic singularities that characterize the IR-regime.

It is known since the early work of Taylor [17] that the IR limit of the ghost-gluon vertex in Landau gauge is not influenced by radiative corrections when an external ghost momentum vanishes. This result is used as the starting point for various IR analyses and is explicitly confirmed by our analysis. In contrast we find a kinematic singularity in the 3-gluon vertex. Although this kinematic singularity which reflects the sensitivity to ultrasoft gluon exchange is rather mild, $\sim\left(p^{2}\right)^{1-2 \kappa}$ with $\kappa \gtrsim 0.5$ [12, 13], it could be conceptually important. First, due to to the parametrically larger support in loop integrals kinematic singularities should have a sizable impact on the quantitative results for Yang-Mills Green functions. Further, it has been argued recently that the Slavnov-Taylor identity for the 3-gluon vertex suggests that the gluon propagator is IR divergent [18. This argument relied on the assumption that the 3gluon vertex is finite when only a single momentum vanishes. Our results show that this is not the case for the scaling solution and thereby the corresponding conclusion cannot be drawn. In addition we show here that the DSEs for the gluonic vertices lead without any approximation or assumption to the condition that the gluon dressing function cannot be IR divergent [15] as argued by Mandelstam [19]. Finally and even more important, the techniques developed here allow one to study the quark sector of QCD, where a corresponding kinematic singularity in the quark-gluon vertex is induced [6]. In contrast to the gauge vertex where the kinematic singularity is induced semi-perturbatively, cf. [20], the one in the quark-gluon vertex is much stronger divergent due to a self-consistent enhancement mechanism. Thereby it induces long ranged gluonic interactions that can confine quarks [6, 7].

In contrast to the assumptions of present scaling analyses the DSE system entails also the possibility of dynamical 


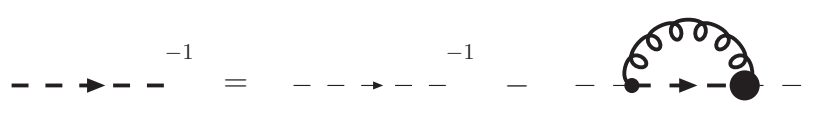

Figure 1: DSE for the ghost-propagator. Thin and thick lines and dots represent bare and proper propagators and vertices. All proper Green functions are amputated and the external lines are shown only for illustration purposes.

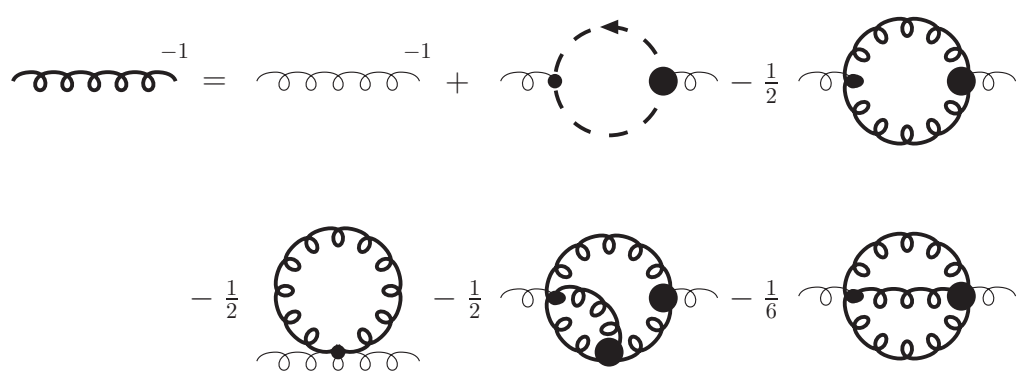

Figure 2: DSE for the gluon-propagator.

mass generation. In addition to the IR scaling solution a decoupling solution where the gluons become massive has recently been suggested [21 23], see also [24, 25]. This solution is also observed in recent lattice simulations [3, 35. Whereas there is only a single scaling solution the decoupling case seems to allow a whole family of solutions characterized by the IR gluon mass that continuously connects with the scaling solution. These different solutions are obtained depending on the chosen boundary conditions for the functional equations [23], cf. also [21]. In case of the decoupling solution certain IR Green functions are not dominated by IR modes but by finite scales of the order of the characteristic scale $\Lambda_{Q C D}$. We extend the power counting formalism in order to take this possibility into account in our IR analysis. Thereby we confirm that the decoupling is compatible with the vertex equations and indeed presents a second solution of the full DSE system. However, we find that within the decoupling solution none of the vertices is IR enhanced. Correspondingly, no long ranged interaction is reflected in the Green functions of the fundamental local degrees of freedom.

This article is closely related to another article [20] on the IR limit of Yang-Mills theory and the behavior of the vertices. There we provide explicit, analytic solutions for the 3-point vertices in a semi-perturbative approximation and confirm and elaborate on the results discussed here.

\section{GENERALIZED INFRARED FIXED POINT ANALYSIS}

In this section we present the general formalism that allows one to study the non-perturbative IR scaling behavior of Green functions. In particular, we take into account that the IR fixed points can be influenced by large scales, like external momenta of Green functions that remain finite in the IR limit and can lead to kinematic singularities, or dynamically generated masses. Our analysis is based on the system of Dyson-Schwinger equations that describe the complete non-perturbative dynamics of the considered theory. The equations for arbitrary vertex functions can be derived algorithmically as shown in [26] and as has been implemented in the Mathematica package DoDSE. The algorithm can be represented by diagrammatic replacement rules applied to the underlying equations for the 1-point functions and is sketched in Appendix A.

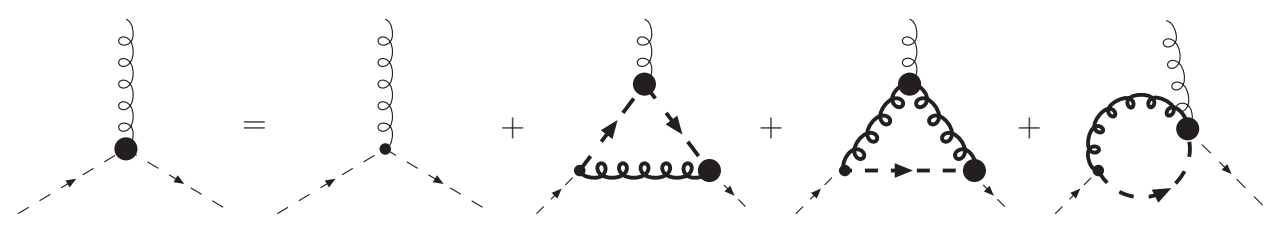

Figure 3: First version of the DSE for the ghost-gluon vertex. The first order in a skeleton expansion consists of the first three diagrams on the right-hand side. The fourth diagram, which features the 1PI ghost-gluon scattering kernel, contributes only at higher order. 

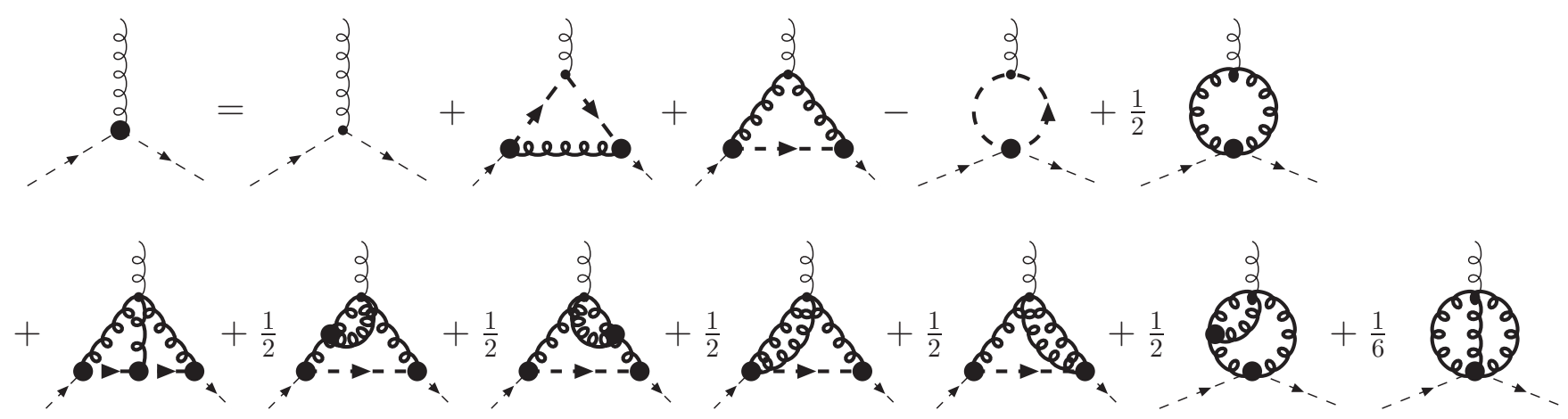

Figure 4: Second version of the DSE for the ghost-gluon vertex. Here the leading order in the skeleton expansion of the right hand side consists of the first three diagrams in the first and second lines, respectively.
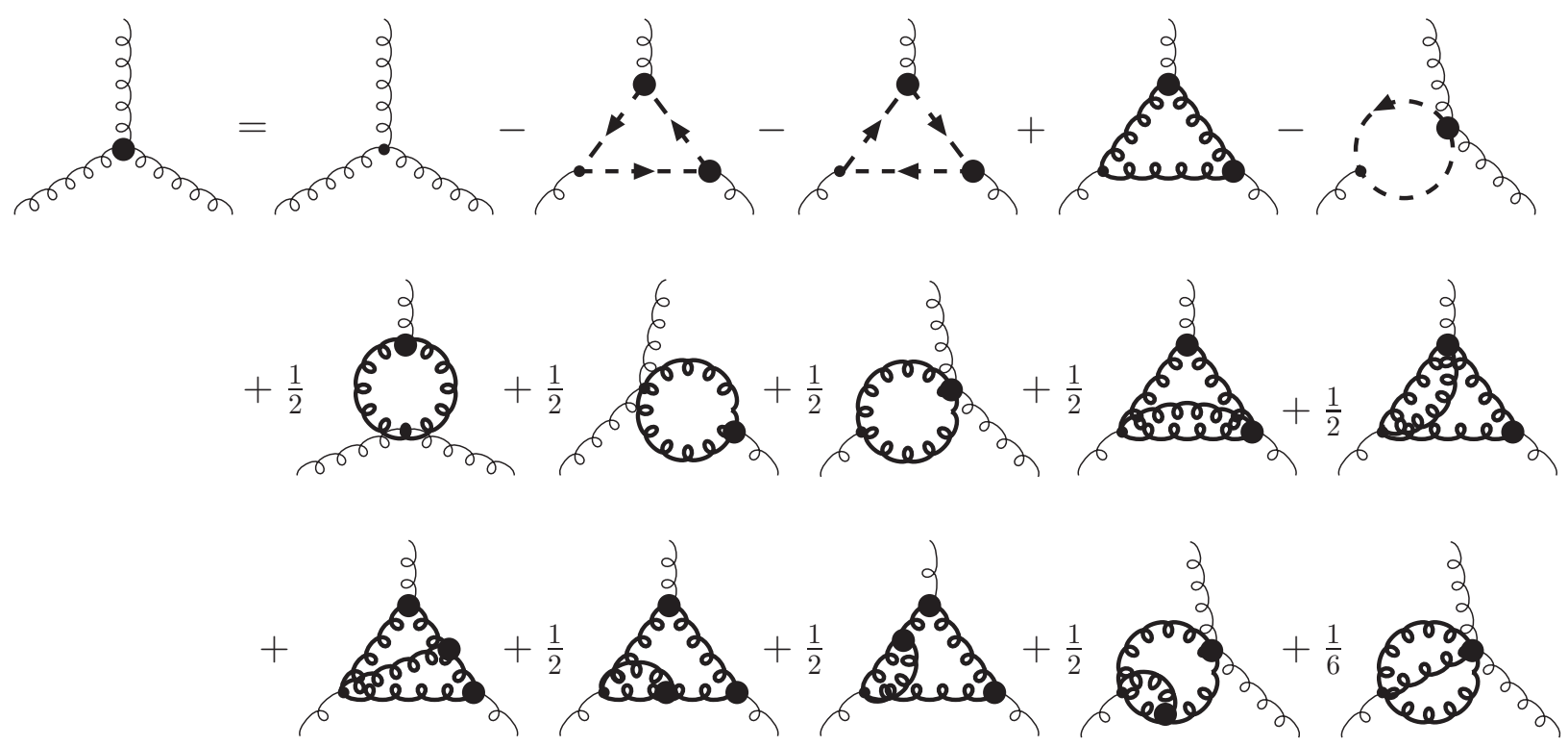

Figure 5: Full DSE for the 3-gluon vertex. To leading order in a skeleton expansion the last graphs in the first respectively third line are absent. The restriction to the 1-loop graphs reduces to the leading order in $\alpha_{s}$ when dressed Green functions are replaced by their tree-level expressions.

The equations for the propagators and primitively divergent vertices of Landau gauge Yang-Mills theory for instance are given in figs. 1 to 6 . As can be seen, the DSEs for the primitively divergent Green functions are in general not closed. Instead they are part of an infinite hierarchy of coupled equations and thereby it might seem hopeless to make any definite statements about existence and uniqueness of possible fixed point solutions. However, as has been demonstrated in [14, this is even possible on the level of a mere IR power counting analysis. The working hypothesis of such an analysis is that in the IR regime the theory can still be described by the field content of the classical action. It involves in the present case as a tool the concept of a skeleton expansion of higher order correlation functions in terms of skeleton diagrams. This means that all vertex functions that do not appear in the Lagrangian are replaced by a loop expansion that features only primitively divergent vertices and propagators. Consequently the system of equations is closed, as it consists only of the DSEs of the primitively divergent vertex functions. The difference compared to a perturbative expansion of these higher vertex functions is, however, that all the primitively divergent Green functions in the expansion loops are dressed. Since the DSEs employed here are based on one-particle irreducible (1PI) vertices, graphs that involve vertices that are not primitively divergent do not contribute to leading order in the skeleton expansion. E. g. for the vertices of Landau gauge Yang-Mills theory the leading order in the skeleton expansion is discussed in the captions of figs. 3 to 6 . The skeleton expansion yields a closed DSE system for the primitively divergent Green functions but involves graphs of arbitrary loop order. In the framework of a power counting analysis it is possible to assess the IR scaling of all these graphs since higher orders can be generated by simple extension 

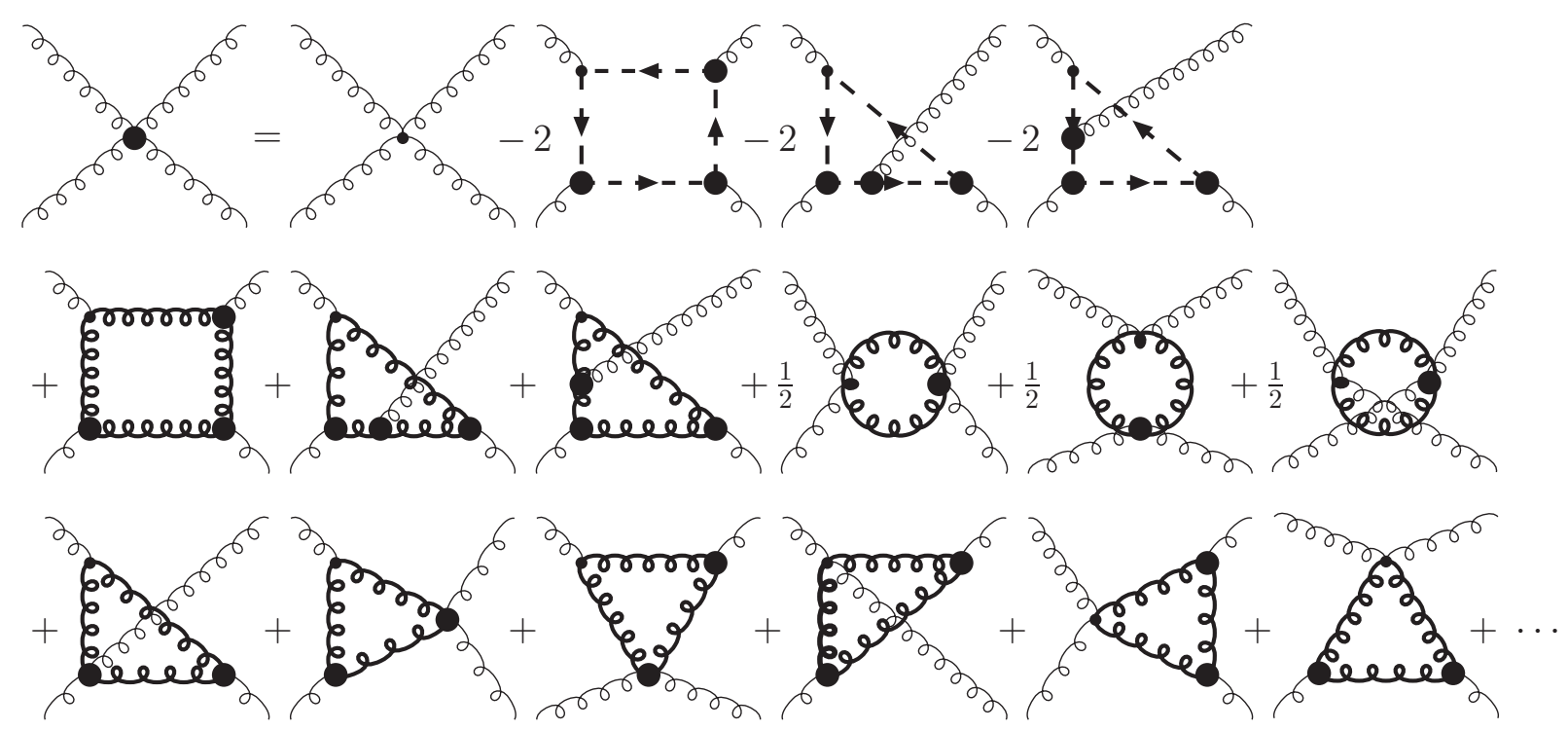

Figure 6: DSE for the 4-gluon vertex - for conciseness we only show the 1-loop contribution in the skeleton expansion. This reduces to the leading order in $\alpha_{s}$ when dressed Green functions are replaced by their tree-level expressions.

rules [14]. As discussed in more detail below, such extensions by additional loops should in particular not increase the degree of IR divergences since higher order diagrams would otherwise be more and more divergent and the description in terms of the underlying degrees of freedom would break down. Correspondingly the lowest order in the expansion already entails in general the leading IR scaling. The solutions obtained with a skeleton expansion are by construction solutions of the full system of DSEs, where the scaling of higher order Green functions is already determined by the leading order skeleton graphs. Note that the skeleton expansion does not miss additional solutions where the nontrivial behavior is triggered by $n$-point functions, $n>4$ [7]. In the case of scaling fixed points the IR analysis has already been performed without the tool of the skeleton expansion in [15, 27. Furthermore, the equivalence between the constraints obtained from the skeleton expansion and constraints derived from functional renormalization group equations has been shown in [27]. Hence the use of the skeleton expansion is well justified.

Whether a solution, where the underlying degrees of freedom are still valid in the IR regime, actually exists can only be decided by an explicit analysis of the non-perturbative dynamics. Once found, a corresponding solution exists then independently of the skeleton expansion and directly verifies the working hypothesis. Yet, our analysis does not exclude other possible solutions where this is not the case. A consequence of our working hypothesis is that there should be no singularities in the external momenta in two- and three-point functions as long as all scales are finite. Such a singularity arises e.g. in the perturbative running of the strong coupling in the form of the Landau pole, but in this case it merely reflects the insufficient dynamical treatment. Yet, in a fully non-perturbative analysis it would signal the breakdown of the description in terms of the fundamental gauge degrees of freedom. In contrast, it is for instance generally expected that the physical degrees of freedom in Yang-Mills theory are glueball bound states which are reflected as singularities in higher gluonic $n$-point functions with $n \geq 4$. These singularities arise at finite invariant momenta corresponding to the masses of the glueball states. In Euclidean space these poles are on the negative half axis of the squared momentum variable and do not interfere with our analysis.

The motivation for an IR analysis is that certain theories, including in particular the important case of gauge theories, are scale invariant at the classical level. Yet, the quantum theory can dynamically generate a scale $\Lambda$ via dimensional transmutation. Whereas e.g. in a perturbative analysis of Yang-Mills theory the corresponding scale $\Lambda_{Q C D}$ is renormalization scheme dependent but otherwise uniquely defined by the Landau pole, non-perturbatively there is no unique definition and it can be chosen as an arbitrary hadronic scale. For the scaling solution of Landau gauge Yang-Mills theory [11, discussed in more detail below, it could for instance be identified with the scale where the gluon propagator reaches its maximum. The leading behavior of all Green functions far below the scale $\Lambda$ should by renormalization group arguments be described by a power law scaling with appropriate IR exponents. Although such a power law form is obtained analytically in conformal field theories and has been observed in scale free regimes of simpler models, for Yang-Mills theory the existence of an IR power law scaling has to our knowledge not been rigorously proven, yet, and presents the main assumption we make in our analysis. To be precise, in this work we determine the IR solutions of Yang-Mills theory that feature IR power law scaling but cannot exclude other possible 
solutions that involve an IR momentum dependence with a more complicated analytic structure. In particular it is conceivable that in addition to a power law scaling there are additional logarithmic dependencies, like it is the case in the UV regime of the theory. We do not explicitly take into account this possibility, but we note that such additional logarithmic dependencies would only refine the scaling and not change the dominant power law structure. Thereby the qualitative results of this analysis remain unaltered.

Whereas the propagators depend only on a single external momentum scale and correspondingly have a unique IR behavior, the vertices involve several momenta and can feature different IR power laws in different kinematic sections. To expose the IR behavior, we perform a tensor decomposition of a given proper Green function $\Gamma_{v}$ with appropriate Lorentz indices $\mu_{i}$ and internal indices $a_{j}$ in some tensor basis $T_{t}$ that is analytic in the momenta

$$
\left(\Gamma_{v}\right)_{\mu_{1} \cdots \mu_{m}}^{a_{1} \cdots a_{n}}\left(q_{1}, \cdots, q_{n}\right)=\sum_{t} \Gamma_{v, t}\left(q_{1}^{2}, q_{1} \cdot q_{2}, \cdots, q_{n}^{2}\right)\left(T_{t}\right)_{\mu_{1} \cdots \mu_{m}}^{a_{1} \cdots a_{n}}\left(q_{1}, \cdots, q_{n}\right)
$$

so that the corresponding dressing functions $\Gamma_{v, t}$ depend only on scalar arguments. Under the assumption of IR scaling, Green functions can in principle have distinct IR power laws whenever any combination of the external momenta vanishes. The dressing functions generally take this power law scaling form only in the deep IR regime $p_{i}^{2} \ll \Lambda$. For intermediate momenta of the order of $\Lambda$ they can involve a more intricate structure and in the UV region they feature a different asymptotic scaling behavior, that is e.g. in Yang-Mills theory given by the perturbative logarithmic RG running. Nevertheless, the full dressing functions can be formally decomposed into a sum of terms that include the individual IR power laws in the different kinematic regimes

$$
\Gamma_{v, t}\left(q_{1}^{2}, q_{1} \cdot q_{2}, \cdots, q_{n}^{2}\right)=\sum_{l} \gamma_{v, t}^{l}\left(q_{1}^{2}, q_{1} \cdot q_{2}, \cdots, q_{n}^{2}\right)\left(\frac{p_{l}^{2}\left(q_{1}^{2}, \cdots, q_{n}^{2}\right)}{\Lambda^{2}}\right)^{\delta_{v, t}^{l}}
$$

In the following we explain this expression in detail. Here, the set of functions $p_{l}^{2}$ defines the scaling variables in the different kinematic limits $l$ which are chosen analytic in all arguments and identical for all Green functions. The $\delta_{v, t}^{l}$ are the corresponding power law exponents. In the IR limit that the scaling variable vanishes, the corresponding function $\gamma_{v, t}^{l}$ in the decomposition eq. 2 is defined such that it depends only on finite momentum ratios

$$
\gamma_{v, t}^{l}\left(q_{1}^{2}, q_{1} \cdot q_{2}, \cdots, q_{n}^{2}\right) \underset{p_{l}^{2} \rightarrow 0}{\longrightarrow} \gamma_{v, t}^{l}\left(\left\{\frac{q_{l_{j}}^{2}}{p_{l}^{2}}\right\}\right)
$$

where the scaling variable $p_{l}$ depends only on the momenta $q_{l_{j}}$ that vanish in the kinematic limit. In particular if the scaling variable depends only on a single external momentum, $\gamma_{v, t}^{l}$ becomes constant in the IR limit. In general, the IR exponents $\delta_{v, t}^{l}$ also depend on the specific tensor. In our analysis we will not distinguish between the different tensor structures and are only interested in the IR exponents of the most singular dressing functions $\delta_{v}^{l} \equiv \min _{t}\left(\delta_{v, t}^{v}\right)$ which will dominate in the IR.

We should stress, that the additive decomposition eq. (2) which explicitly separates the different IR divergences of a dressing function into different summands does not represent an IR approximation and is exact on all momentum scales. Yet, it is surely far from unique. This ambiguity is reflected in the functions $\gamma_{v, t}^{l}$ which include the proper mid and high momentum behavior and also depend on the choice of the arbitrary scale $\Lambda$. This means that the explicit IR power law is compensated in the functions $\gamma_{v, t}^{l}$ outside of the IR region. As discussed above, for $n$-point functions with $n \geq 4$ they may involve singularities when all combinations of external scales are finite but they are by definition regular whenever external scales vanish. The only requirement on the partition is the complete decomposition of possible IR singularities. For the determination of the IR power law exponents, however, the detailed form of the decomposition at finite scales is irrelevant as discussed below.

An important IR limit is determined by a uniform scaling variable that is given by a function $p_{u}$ that fulfills

$$
p_{u}^{2}\left(q_{1}^{2}, \cdots, q_{n}^{2}\right) \rightarrow 0 \quad \Leftrightarrow \quad q_{1}, \cdots, q_{n} \rightarrow 0 \wedge \quad q_{1}^{2} / p_{u}^{2}, \cdots, q_{n}^{2} / p_{u}^{2} \quad \text { constant . }
$$

Such a function is for instance provided by the "Euclidean norm"

$$
p_{u}^{2}\left(q_{i}^{2}\right) \equiv \sum_{i} q_{i}^{2}
$$




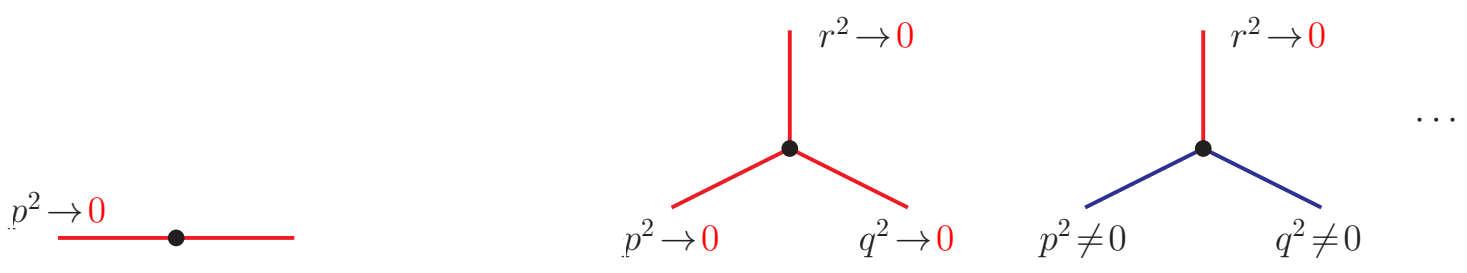

Figure 7: The unique IR singular kinematic configuration of the propagator (left) and the different IR singular kinematic configurations of the 3-point functions (right).
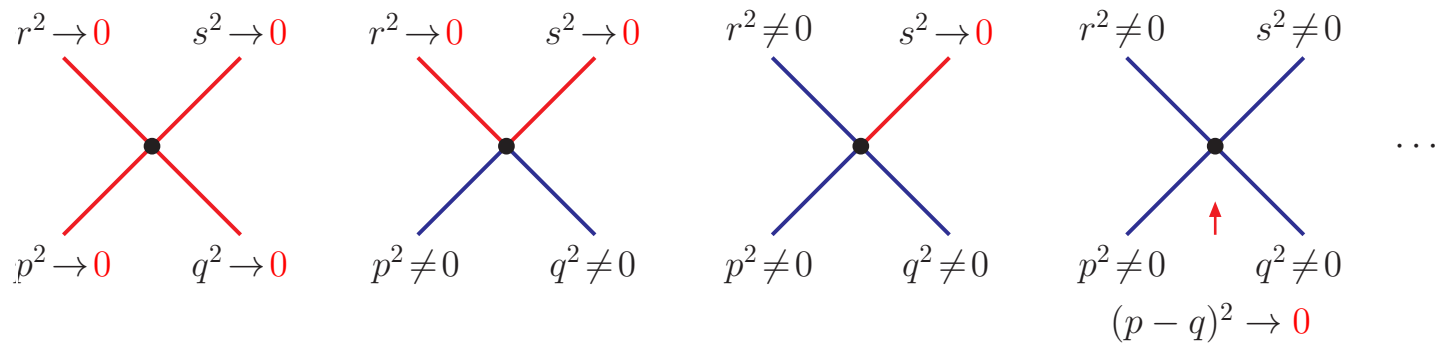

Figure 8: IR singular kinematic configurations of the 4-point functions. In this case there can even be kinematic singularities when all external momenta are hard.

Since in this scaling limit all external momenta scale uniformly to zero this presents the fully scale-invariant case. Previous studies assumed that all Green functions are finite in other kinematic limits and thereby have only considered this "conformal"1 case [14 16]. We will discuss this important approximation in section IV.

In general, however, there are various different kinematic limits where the $p_{l}$ can also depend on any subset of the $q_{i}$. As we will argue below in section V, in Yang-Mills theory such a more general IR behavior is realized that involves kinematic singularities according to eq. (2) when only a subset of the external momenta vanishes. We will denote those external momenta that scale to zero as soft and those that stay fixed when the IR limit is taken as hard momenta. In particular, all soft external momenta are assumed to be much smaller than $\Lambda$ in order to ensure the applicability of IR power laws. By momentum conservation it is impossible that exactly $n-1$ momenta of an $n$-point function become soft, but e.g. all other cases when $1 \leq i \leq n-2$ momenta tend to zero can define a separate scaling limit. The simplest such kinematic limit is when a single external momentum becomes soft and all others remain hard where the corresponding scaling variable in eq. (2) is $p_{i}^{2} \equiv q_{i}^{2}$. For each distinct external field this presents a different limit described by a separate power law exponent. These present the only kinematic limits in case of 3-point functions, as illustrated in fig. 7. As shown in fig. 8, for the 4-point functions there are already two distinct limits when one or two external legs are soft. In addition, there can even be IR singularities for 4-point functions when all external momenta are hard but differences of momenta become small corresponding to the exchange of a soft momentum in the respective intermediate channel. For higher order Green functions the number of IR exponents rises further.

Now let us discuss how to determine the IR behavior of the integrals arising from the loop corrections in the DSEs. Inserting the parameterization eq. (2) into a general 1-loop integral $\mathcal{G}$ yields a sum of different integrals of the form

$$
\mathcal{G}=\prod_{v} \sum_{l_{v}} \Lambda^{-2\left(\sum_{v}\left(\delta_{v}+\delta_{v}^{l v}\right)\right)} \int \frac{d^{d} k}{(2 \pi)^{d}} \mathcal{K}_{\left\{l_{v}\right\}}\left(\left\{(k+Q)^{2}\right\},\left\{Q^{2}\right\}\right) \prod_{m}\left(k+Q_{m}\right)^{2\left(\delta_{m}-1\right)} p_{l_{v}}^{2}\left(\left\{(k+Q)^{2}\right\},\left\{Q^{2}\right\}\right)^{\delta_{v}^{l_{v}}}
$$

where $m$ and $v$ run over the internal propagators and vertices in the loop and $l_{v}$ over the the different IR sensitive limits of the vertex $v$. The $Q_{i}$ are linear combinations of the external momenta and the functions $\gamma_{v}^{l_{v}}$ as well as any other analytic terms arising from the tensor decomposition are combined in the kernels $\mathcal{K}_{\left\{l_{v}\right\}}$. They may involve additional power law divergences in the external momenta alone but are analytic in the loop momentum. Such integrals arise in addition to the explicitly shown IR sensitive kinematical cases also for the individual terms from the tensor decomposition eq. (11). We note in particular that due to the scaling form eq. (2) the dependence on the scale

\footnotetext{
1 The term conformal refers in our context only to the scale invariance property but not the additional symmetries included in the conformal group.
} 
$\Lambda$ factors out of the actual integral.

The scalar integrals that remain after an appropriate tensor decomposition are in general far too complicated to be performed analytically. However, in the special case that the vertices are constant explicit solutions are known. The 1-loop two-point integrals are given by the simple analytic form

$$
\int \frac{d^{d} k}{(2 \pi)^{d}}\left(k^{2}\right)^{\nu_{1}}\left((k-q)^{2}\right)^{\nu_{2}}=(4 \pi)^{-\frac{d}{2}} \frac{\Gamma\left(\frac{d}{2}+\nu_{1}\right) \Gamma\left(\frac{d}{2}+\nu_{2}\right) \Gamma\left(-\frac{d}{2}-\nu_{1}-\nu_{2}\right)}{\Gamma\left(-\nu_{1}\right) \Gamma\left(-\nu_{2}\right) \Gamma\left(d+\nu_{1}+\nu_{2}\right)}\left(q^{2}\right)^{\frac{d}{2}+\nu_{1}+\nu_{2}}
$$

for $d / 2+\nu_{1}+\nu_{2} \leq 0, d / 2+\nu_{1} \geq 0$ and $d / 2+\nu_{2} \geq 0$, where the expression is convergent. An analytic expression for the corresponding IR 3-point integrals in terms of hypergeometric functions is known [28, 29] and we discuss how to extend this result to the Euclidean regime of these integrals in [20].

However, in order to solely determine the IR scaling of a given integral an explicit solution is not required. In the limit that some external scales tend to zero and others stay finite one has a clear scale separation. As is well known from effective field theories and shown in detail in appendix B this allows one to effectively decouple these scales. The basic steps are pictorially illustrated in fig. 9. An integral involving both hard and soft scales is first divided at an arbitrary intermediate scale. The now limited support of the two integrals allows one to Taylor-expand the integrand so that in the IR limit, where the lowest order is exact, the individual integrals depend only on either soft or hard scales and the respective other scales factor out of the integral. Next, the two integrals are extended over the whole scale range again. This leaves two correction terms that can be expanded once more and combined to a scale-independent integral extending over all scales. It merely presents a counterterm to cancel possible divergences introduced in the process of separating the initial integral. Thereby the initial integral is effectively decomposed into two separate integrals that depend only on one of the two classes of scales respectively, whereas in the soft part the dependence on the hard scales factors out of the integral. On dimensional grounds such integrals must scale as a function of this single scale and the power law exponents can then be determined by a mere power counting analysis. This works since the integrals are effectively dominated by the poles of the integrand that are in turn determined by the external momenta. In particular, these distinct integrals can each feature a different IR scaling behavior. Thereby, when assessing the IR scaling of a given correlation function via its DSE, all these partial integrals over the different kinematic regions of the initial loop integral can be analyzed individually and the most IR divergent term determines the IR scaling. As discussed in appendix B, it can be necessary to repeat this decomposition when multiple soft and hard scales are present. After a complete decomposition the different kinematic contributions of the initial integral can be displayed and treated just as distinct Feynman graphs, as shown in fig. 10 for the soft-particle limit of the 3 -point function, where the momenta of the internal propagators in the individual contributions are restricted to be soft $(s)$ respectively hard $(h)$ as indicated.

Furthermore, it is necessary to consider the presence of mass scales in the IR analysis. In addition to the case of theories that have a sector which involves explicit mass scales at the classical level which is coupled to a conformal sector, like QCD, there is even the possibility of dynamical mass generation in theories that are scale-free at the classical level, like Yang-Mills theory studied below. In general a massive behavior of a (scalar) propagator $D$ is defined by

$$
\lim _{p \rightarrow 0} D\left(p^{2}\right)=\text { const }
$$

This yields for the corresponding IR exponent $\delta$

$$
D\left(p^{2}\right)=\frac{Z\left(p^{2}\right)}{p^{2}} \underset{p \rightarrow 0}{\longrightarrow} \frac{p^{2 \delta}}{p^{2}} \sim p^{0} \Rightarrow \delta=1 .
$$

In this case the IR scaling is broken since an explicit mass scale

$$
m^{2} \equiv \lim _{p \rightarrow 0} D^{-1}\left(p^{2}\right)
$$

is present and the propagator can be parameterized e.g. by the alternative forms

$$
D\left(p^{2}\right)=\frac{\tilde{Z}\left(p^{2}\right)}{p^{2}+m^{2}}=\frac{1}{p^{2}+M^{2}\left(p^{2}\right)} \quad \text { with } \quad \tilde{Z}\left(p^{2}\right) \equiv \frac{p^{2}+m^{2}}{p^{2}} Z\left(p^{2}\right), \quad M\left(p^{2}\right) \equiv p^{2}\left(\frac{1}{Z\left(p^{2}\right)}-1\right)
$$




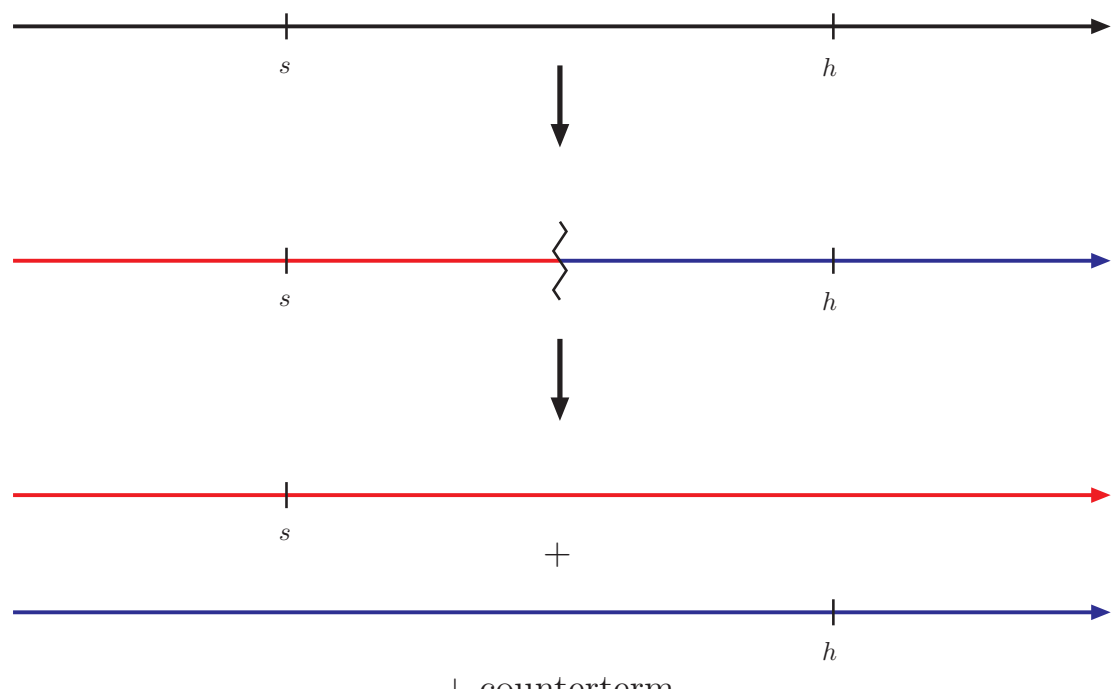

Figure 9: Pictorial representation of the decomposition of loop integrals described in detail in appendix B A loop integral that involves both a soft $s$ and a hard scale $h$ can in the limit $s \ll h$ be divided at an arbitrary intermediate scale and decomposed into two independent loop integrals and a counterterm that cancels possible divergences introduced in this process. Each of these two integrals involves only a single scale whereas the other scale factors out of the corresponding integral. This allows a direct power counting of the IR scaling behavior of the individual contributions.
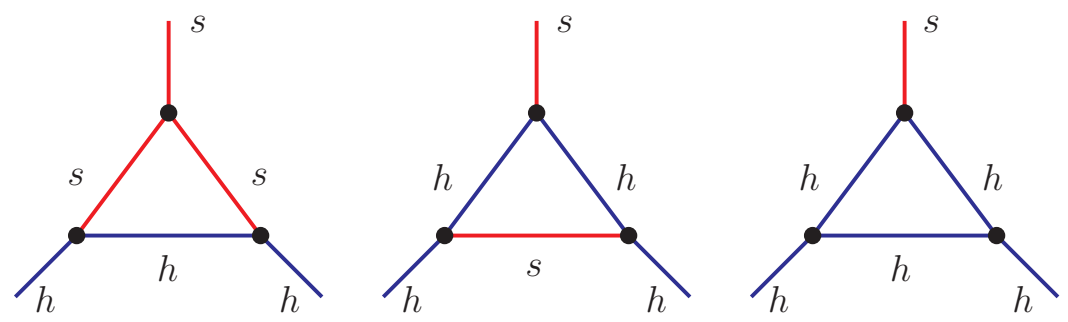

Figure 10: IR singular kinematic regions in the decomposition of the triangle integrals contributing to the 3-point functions in the soft particle limit. There are two different kinematic regions that involve soft loop momenta corresponding to the two inequivalent ways to route the hard momentum through the loop.

In this form it is clear that integrals can also be dominated by scales of the order of the mass, as discussed in detail in appendix $\mathrm{B}$. In the presence of mass scales there are relevant contributions from corresponding hard momentum scales in the loop integration even in the case that all external scales are soft, as shown in fig. 11. If these hard contributions dominate such a dynamically generated mass presents a self-consistent solution of the DSE system and poses an additional possibility for the IR behavior that has to be taken into account.

In the uniform limit the scaling is very different in the massive and massless case. When there are only massive propagators in a loop integral the integral is entirely dominated by hard scales even in the uniform limit and does not scale with the external soft scales, see appendix B. When only some propagators are massive the integral can again be decomposed and receives contributions from both soft and hard momentum regions, as is again shown in appendix B and is depicted in fig. 11. In contrast, in the massless case the integrals are IR dominated in the uniform limit and the contribution from hard loop momenta is strongly suppressed. This can be seen by expanding the analytic functions $\mathcal{K}_{\left\{l_{v}\right\}}$ in eq. [6] as well as the arising propagators in the limit $q_{i} \ll k$, where $k$ is the loop momentum, 

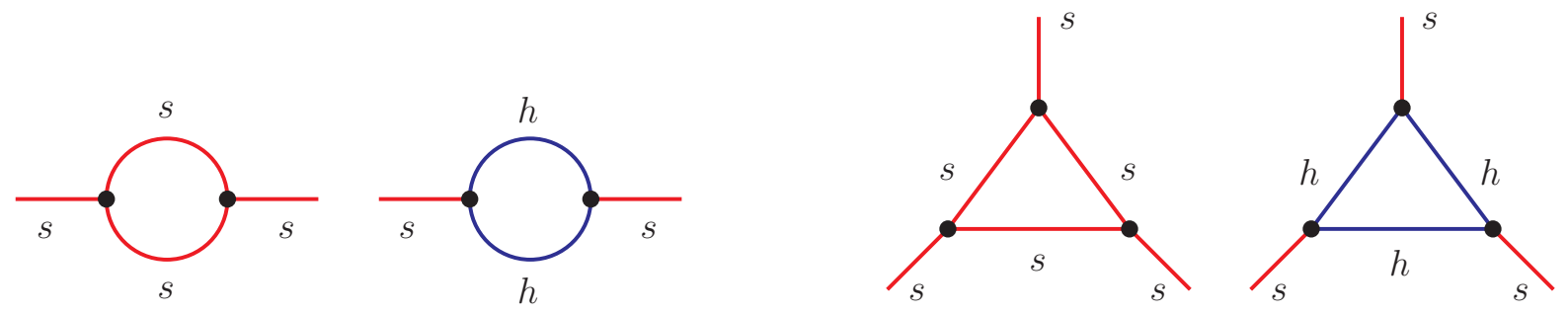

Figure 11: Possibly dominant kinematic regions of 1-loop integrals of the propagator (left) and the 3-point function in the uniform limit (right). The labels $s$ and $h$ denote soft momenta that vanish when the scaling variable tends to zero and hard momenta that stay finite in the limit, respectively.

$$
\begin{aligned}
\frac{1}{\left(k+q_{i}\right)^{2(1-\alpha)}} & =\frac{1}{k^{2(1-\alpha)}}\left(1+2 \frac{q_{i} \cdot k}{k^{2}}+\frac{q_{i}^{2}}{k^{2}}\right)^{\alpha-1} \\
& \approx \frac{1}{k^{2(1-\alpha)}}\left(1+2(\alpha-1) \frac{q_{i} \cdot k}{k^{2}}+(\alpha-1) \frac{q_{i}^{2}}{k^{2}}+2(\alpha-1)(\alpha-2) \frac{\left(q_{i} \cdot k\right)^{2}}{k^{4}}+O\left(\frac{q_{i}^{3}}{k^{3}}\right)\right)
\end{aligned}
$$

The leading order term is indeed independent of the external momenta. In case there are no other scales involved, i.e. in particular $\alpha \neq 1$, cf. appendix $B$ this scale independent integral as well as the third term is canceled or removed in the renormalization process, as discussed below. The linear term vanishes likewise in the symmetric integration so that only the last term remains and the contribution from hard modes is actually suppressed in $q_{i}^{2} / k^{2}$. Yet, when there is any explicit scale in the integral, like a hard external momentum or a mass (corresponding to $\alpha=1$ ), the leading term in the expansion eq. $\sqrt{12}$ is not canceled and there is no suppression. In order to treat the different cases within a common framework and allow for all possible solutions, including those where masses are dynamically generated, we count in all cases the contributions from both the hard and soft momentum region of the loop integral. In case a mass is generated dynamically it arises from quantum fluctuations in the "mid-momentum regime", i.e. hard scales of the order of the generated mass. To establish whether a massive solution actually exists and to obtain a quantitative value for the mass requires a numerical solution of the functional equations for all momenta. However, a mere IR analysis can nevertheless determine if a dynamically generated mass presents a possible dynamical solution of the DSE system. This is the case since the above expansion eq. 12 shows that the different cases of a massless and a massive propagator are effectively discriminated by their IR exponents. In the power counting study below we implement this qualitatively different scaling of the propagators arising in loop integrals via the symbol $\mu_{i}$ where the index $i$ stands for the corresponding particle species and which is defined by

$$
\mu_{i}=\left\{\begin{array}{lll}
0 & \text { for } & \delta_{i}=1 \\
1 & \text { for } & \delta_{i} \neq 1
\end{array}\right. \text {, i.e. a massive IR behavior }
$$

Since eq. (12) shows that the contribution from the hard momentum regime of a loop integral is suppressed only if all propagators are massless, the suppression of the whole loop integral is then given by adding the product of the $\mu_{i}$ of all propagators in the loop to the loop's IR exponent. We stress that although in the considered region of hard momenta the dressing functions do not feature the IR scaling form, the above prescription is meaningful for the power counting analysis since the massive behavior is nevertheless reflected in the anomalous IR power laws by the IR exponent $\delta_{i}=1$.

The loop integrals in the DSEs are UV divergent and must be renormalized. Since the leading Green functions have positive mass dimension power law divergences seem to pose a threat to a naive power counting analysis because hard momenta could contribute even in the case that all external momenta are soft. Actually, the above suppression explicitly shows the known fact that all power law divergences, like e.g. the quadratic divergences in the propagators, are momentum independent in scale invariant theories. If present, these identically cancel between the different loop graphs in a DSE. Whereas this is generally guaranteed by the perturbative UV behavior so that the contributions from asymptotically large momenta $k \gg \Lambda$ to the integral cancel, in the IR scaling limit the cancellation of contributions from hard modes is according to eq. (12) already realized for loop momenta $k \gg p$. Similarly there are dedicated counterterms that by construction explicitly cancel any additional singularities introduced in the above decomposition, cf. appendix B. This leaves at most logarithmic momentum-dependent divergences to be removed within the 
renormalization procedure. As shown e.g. in [11, 13, this is possible multiplicatively by corresponding wave-function and vertex renormalization factors that multiply the corresponding tree level terms as well as the loop corrections in the DSEs. Since the logarithmic momentum-dependence is not considered within the power counting analysis anyhow, it is clear that the problem of renormalization does not interfere with the IR scaling analysis as could have been expected considering that they operate in opposite limits of the loop integrals. In principle the analysis can also be performed directly for already subtracted DSEs where the issue is omitted from the outset. Thereby, in general UV divergences and the detailed renormalization procedure do not affect the IR analysis. Yet, there is one important exception to this since under certain conditions discussed below the renormalization prescription can determine the boundary conditions for the DSE system 23 .

After a complete decomposition of the loop integrals in the DSEs, the IR scaling of each contribution is determined via power counting of the anomalous and canonical dimensions of the involved Green functions. The most divergent contribution - corresponding to the minimal IR exponent - dominates and determines the scaling of the Green function on the left hand side of the DSE. Finally it is important to note that there can be various cancelations caused by the symmetries of the considered theory that require a careful analysis. This will be discussed in detail in section $\mathrm{V}$ for the case of Landau gauge Yang-Mills theory.

\section{DISPROVAL OF THE NAIVE INFRARED SLAVERY SCENARIO}

After this general section we will now begin our IR analysis of Yang-Mills theory. The equations for the propagators of Landau gauge Yang-Mills are given in figs. 1 and 2. These equations have been studied extensively with appropriate ansaetze for the vertices. The corresponding DSEs for the primitively divergent vertex functions of Yang-Mills theory are given in figs. 3 to 6 . For the ghost-gluon vertex there are two qualitatively different versions derived via the ghostrespectively gluon-part of the path integral. The leading order in a skeleton expansion is given by the two triangle diagrams that have been analyzed in [30] within a semi-perturbative analysis, where it was found that this vertex is hardly changed from its tree-level form. The other vertices have so far been discussed only via IR scaling analyses [14 16] which we will detail in this work. Explicit IR results for the 3-point vertices are given in [20].

Before we study the implications of the system of equations for the IR limit of Yang-Mills theory, we will demonstrate the power counting method explicitly for an important example. Consider the first two diagrams of the second line in the DSE for the 3-gluon vertex fig. 5 The contribution from these two graphs to the right hand side of the DSE is

$$
\begin{aligned}
\Delta \Gamma_{\mu \nu \rho}^{a b c}\left(q_{1}, q_{2}\right)=\frac{1}{2} \int \frac{d^{4} k}{(2 \pi)^{4}} & \left(\left(\Gamma_{0}\right)_{\rho \mu \alpha \beta}^{c a d e} D_{\alpha \gamma}^{d f}\left(k-q_{2}\right) \Gamma_{\gamma \delta \nu}^{f g b}\left(k-q_{2},-k, q_{2}\right) D_{\delta \beta}^{g e}(k)\right. \\
& \left.+\left(\Gamma_{0}\right)_{\nu \rho \alpha \beta}^{b c d e} D_{\alpha \gamma}^{d f}\left(k-q_{1}\right) \Gamma_{\gamma \delta \mu}^{f g a}\left(k-q_{1},-k, q_{1}\right) D_{\delta \beta}^{g e}(k)\right),
\end{aligned}
$$

where $D$ represents the dressed gluon propagator, $\Gamma$ the dressed vertices distinguished by their indices, and $\Gamma_{0}$ the bare versions. We note that each of the two integrals depends on only one of the two independent external momenta, because the bare 4-gluon vertex is momentum independent. Therefore, the two integrals cannot exactly cancel each other for general momenta. With the uniform IR scaling exponents $\delta_{g l}$ for gluon propagators and the corresponding uniform exponent $\delta_{3 g}^{u}$ for the 3 -gluon vertex each of the integrals as well as its sum scales in the uniform limit as

$$
\Delta \Gamma_{\mu \nu \rho}^{a b c}\left(q_{1}, q_{2}\right) \underset{p^{2} \rightarrow 0}{\longrightarrow} p^{4}\left(\left(p^{2}\right)^{-1+\delta_{g l} l}\right)^{2}\left(p^{2}\right)^{\frac{1}{2}+\delta_{3 g}^{u}} \sim\left(p^{2}\right)^{\frac{1}{2}+\delta_{3 g}^{u}+2 \delta_{g l}} .
$$

We see that the canonical scaling of the 3-gluon vertex given by the $\frac{1}{2}$ drops out since it appears both on the left and right hand side of the DSE and as expected it is sufficient to count only anomalous IR exponents in the uniform scaling limit. Each of the contributions on the right hand side of the DSE - or several of them - could dominate and determine the scaling of the 3-gluon vertex on the left hand side of the DSE. The leading term is the one with the smallest IR exponent. Correspondingly, the IR exponent of the two exemplary graphs has to be larger or equal to the left hand side in case they dominate. This leads to the very general condition

$$
\delta_{3 g}^{u} \leq \delta_{3 g}^{u}+2 \delta_{g l} \quad \Rightarrow \quad \delta_{g l} \geq 0 .
$$

The only other possibility would be that the two integrals are canceled identically by other diagrams in the DSE which correspondingly would have to have precisely the same kinematic dependence. We pointed out before that the two graphs have a very special kinematic structure given by 


$$
\Delta \Gamma_{\mu \nu \rho}^{a b c}\left(q_{1}, q_{2}\right)=F_{\mu \nu \rho}^{a b c}\left(q_{1}\right)+G_{\mu \nu \rho}^{a b c}\left(q_{2}\right)
$$

Evidently all other graphs involve the two momenta in a manifestly non-linear way - e.g. already the propagators in the loop induce manifest non-linearities - and thereby cannot have the above simple property. For instance consider the case of the third gluon loop graph that includes the proper 4-gluon vertex. Its integral representation is

$$
\frac{1}{2} \int \frac{d^{d} k}{(2 \pi)^{d}}\left(\Gamma_{0}\right)_{\mu \delta \alpha}^{a e d}\left(q_{1}, k,-k-q_{1}\right) \frac{Z\left(k+q_{1}\right)}{\left(k+q_{1}\right)^{2}}\left(\delta_{\alpha \beta}-\frac{\left(k+q_{1}\right)_{\alpha}\left(k+q_{1}\right)_{\beta}}{\left(k+q_{1}\right)^{2}}\right) \Gamma_{\nu \rho \beta \gamma}^{b c d e}\left(q_{2},-q_{1}-q_{2}, k+q_{1},-k\right) \frac{Z(k)}{k^{2}}\left(\delta_{\gamma \delta}-\frac{k_{\gamma} k_{\delta}}{k^{2}}\right)
$$

where the gluon dressing functions $Z$ generally involve non-integer powers. Even in the simplest case $\delta_{g l}=1$ where the propagators become trivial the above property would be in contrast to the 1PI nature of the proper 4-point vertex. Since the momentum dependence of the other contributions in the DSE is even more non-linear it is fair to conclude that there are no identical cancelations between the individual diagrams in fig. 5. This yields the direct constraint that the gluon dressing function cannot be singular as found in the Mandelstam approximation [19]. The same relation is obtained from the gluon-loop corrections given by the last three diagrams in the second line of the 4-gluon vertex DSE fig. 6. Note that this result is a direct prediction of the full vertex DSEs and does not involve any assumption or approximation. Moreover, it is independent of the detailed renormalization prescription used for the DSEs. Interestingly, all studies that found an IR enhanced gluon propagator made uncontrolled assumptions on the form of the 3-gluon vertex, whereas such a non-enhanced IR behavior of the propagator was indeed found as soon as the vertex equations were considered dynamically within simplified semi-perturbative analyses, cf. [10].

\section{CONFORMAL SCALING}

In this section we will perform an IR fixed point analysis for Landau gauge Yang-Mills theory but will first neglect the possibility of kinematic singularities or dynamically generated mass scales. Thereby we consider the idealized, conformal case that all external momenta as well as the considered correlation functions scale with a single uniform scaling variable $p_{u}$, as defined in eq. (4), and are finite in other kinematic limits. In this case eq. (2) reduces to

$$
\Gamma_{v}\left(q_{1}^{2}, q_{1} \cdot q_{2}, \cdots, q_{n}^{2}\right) \approx \gamma_{v}^{u}\left(q_{1}^{2}, q_{1} \cdot q_{2}, \cdots, q_{n}^{2}\right) \cdot\left(\frac{p_{u}^{2}\left(q_{1}^{2}, \cdots, q_{n}^{2}\right)}{\Lambda_{Q C D}^{2}}\right)^{\delta_{v}^{u}}
$$

Correspondingly, we neglect the possibility that contributions from hard loop momenta are relevant in the IR limit and assume that only the IR regime dominates all loop integrals. As will be shown in the next section this assumption is too simplified and the solution structure obtained in this section is incomplete.

Since the Dyson-Schwinger equations form an infinitely coupled system of equations it is necessary to reduce it to a manageable form, as discussed in sec. II. This is done via a skeleton expansion that presents a loop expansion in terms of dressed primitively divergent correlation functions. The skeleton expansion can be generated from the leading order graphs by a finite set of extensions that increase their loop order [14] as shown in fig. 12. As a necessary condition for the skeleton expansion these extensions must not increase the IR exponents for a given Green function since otherwise successive extensions would make it arbitrary singular. Thereby, assuming that the skeleton expansion is not explicitly divergent, provides additional constraints for the IR exponents of the primitively divergent vertices. When there is only a single uniform scaling variable $p_{u}$, so that all external momenta scale with it, $q_{i}^{2} \sim p_{u}^{2}$, up to possible subleading corrections an integral has to scale as a power of it (or possibly as a logarithm in case the naive power counting yields a constant). As noted before, in four dimensions all canonical momentum dependence cancels and it turns out that it suffices to count anomalous powers of $p_{u}^{2}$ to assess the IR behavior of a general loop correction. The leading dynamical contribution on the right hand side of its DSE determines the scaling of a given Green function. With the corresponding uniform IR exponents for the ghost propagator $\delta_{g h}$, the gluon propagator $\delta_{g l}$, the ghost-gluon vertex $\delta_{g g}$, the 3 -gluon vertex $\delta_{3 g}$ and the 4 -gluon vertex $\delta_{4 g}$ we can analyze the IR scaling limit of the DSE system for the five primitively divergent Green functions. Here we skip the superscript $u$ on the uniform exponents as it appears in eq. $\sqrt{19}$ for better readability in this section. From figs. 1 and 2 one reads off the power counting relations for the IR exponents of the propagators ${ }^{2}$

\footnotetext{
2 The tadpole contribution in the gluon DSE does not appear here, since it does not depend on the external momentum.
} 


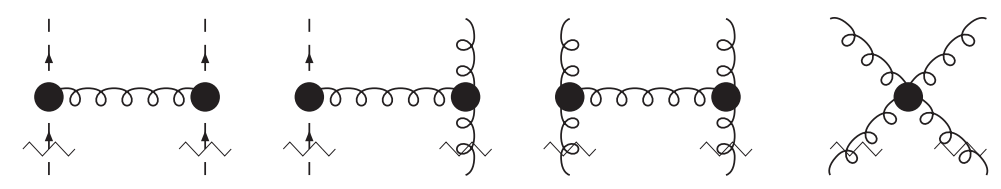

Figure 12: Possible extensions of given graphs to generate higher orders in the skeleton expansions. The crossed out propagators are part of the initial graph and are not counted.

$$
\begin{aligned}
-\delta_{g h} & =\min \left(0, \delta_{g g}+\delta_{g h}+\delta_{g l}\right) \\
-\delta_{g l} & =\min \left(0, \delta_{3 g}+2 \delta_{g l}, \delta_{g g}+2 \delta_{g h}, 2 \delta_{3 g}+4 \delta_{g l}, \delta_{4 g}+3 \delta_{g l}\right)
\end{aligned}
$$

and correspondingly for the vertex functions from figs. 3 to 6

$\delta_{g g}=\min \left(0,2 \delta_{g g}+2 \delta_{g h}+\delta_{g l}, \delta_{3 g}+\delta_{g g}+\delta_{g h}+2 \delta_{g l}\right)$,

$\delta_{g g}=\min \left(0,2 \delta_{g g}+2 \delta_{g h}+\delta_{g l}, 2 \delta_{g g}+\delta_{g h}+2 \delta_{g l}, 3 \delta_{g g}+2 \delta_{g h}+3 \delta_{g l}, \delta_{3 g}+2 \delta_{g g}+\delta_{g h}+4 \delta_{g l}\right)$

$\delta_{3 g}=\min \left(0,2 \delta_{g g}+3 \delta_{g h}, 2 \delta_{3 g}+3 \delta_{g l}, \delta_{3 g}+2 \delta_{g l}, \delta_{4 g}+2 \delta_{g l}, 3 \delta_{3 g}+5 \delta_{g l}, \delta_{4 g}+\delta_{3 g}+4 \delta_{g l}\right)$,

$\delta_{4 g}=\min \left(0,3 \delta_{g g}+4 \delta_{g h}, 3 \delta_{3 g}+4 \delta_{g l}, \delta_{4 g}+2 \delta_{g l}, 2 \delta_{3 g}+3 \delta_{g l}, \delta_{4 g}+\delta_{3 g}+3 \delta_{g l}, 4 \delta_{3 g}+6 \delta_{g l}, \delta_{4 g}+2 \delta_{3 g}+5 \delta_{g l}, 2 \delta_{4 g}+4 \delta_{g l}\right)$.

Besides the constraint $\delta_{g l} \geq 0$ obtained before there are additional analogous constraints from the linear terms in the vertex equations (21). These constraints are weaker than those obtained from the corresponding RG equations studied in [15, 27. and are not sufficient to ensure a unique solution of the system of equations. Therefore, we will not exploit them in the following and thereby circumvent the problem of possible cancelations discussed above.

In its present form the nonlinear system of the DSE conditions (20) and (21) is rather complicated due to the involved minimum functions. However, if one requires that there should exist a stable skeleton expansion, the extension graphs in fig. 12 yield the additional much stronger constraints

$$
\begin{aligned}
2 \delta_{g g}+2 \delta_{g h}+\delta_{g l} & \geq 0, \\
\delta_{3 g}+\delta_{g g}+\delta_{g h}+2 \delta_{g l} & \geq 0, \\
2 \delta_{3 g}+3 \delta_{g l} & \geq 0, \\
\delta_{4 g}+2 \delta_{g l} & \geq 0 .
\end{aligned}
$$

The combination of IR exponents in the first two of these constraints is precisely the one arising from the two triangle diagrams in the first equation for the exponent of the ghost-gluon vertex eq. (21), so that this equation becomes unique whereas the second one remains non-trivial

$$
\delta_{g g}=0 \wedge \delta_{g g}=\min \left(0,2 \delta_{g g}+2 \delta_{g l}+\delta_{g h}\right) .
$$

Since both of these equations have to hold simultaneously it is clear that the second equation has the trivial solution, too. To see that it is not important which DSE we start with, consider only the second equation and assume that the second term dominates. In this case the ghost-gluon vertex would be given by $\delta_{g g}=-2 \delta_{g l}-\delta_{g h}$. Plugging this in the first constraint from the skeleton expansion, eq. $(22)$, yields $\delta_{g l} \leq 0$. Since we know from section III that $\delta_{g l}$ is non-negative, only the trivial solution $\delta_{g l}=\delta_{g h}$ remains.

This non-renormalization condition has previously been used as a starting point in the analysis [14]. It was supported by the gluon transversality in Landau gauge but required the additional assumption that the ghost-gluon scattering kernel should not be strongly divergent. Here it arises directly from the physical requirement of a stable skeleton expansion. The two other constraints in (22) precisely remove the non-linearities in the equations (21) for the gluon vertices which could lead to a self-consistent enhancement of these equations. Inserting this IR scale independence of the ghost-gluon vertex into the other equations and using the constraints eqs. 22 as well as the previously shown relation $\delta_{g l} \geq 0$ yields 


$$
\begin{aligned}
-\delta_{g h} & =\min \left(0, \delta_{g h}+\delta_{g l}\right), \\
-\delta_{g l} & =\min \left(0,2 \delta_{g h}\right), \\
\delta_{3 g} & =\min \left(0,3 \delta_{g h}\right), \\
\delta_{4 g} & =\min \left(0,4 \delta_{g h}, 3 \delta_{3 g}+4 \delta_{g l}\right) .
\end{aligned}
$$

As it stands the naive system of equations above has only the trivial solution that all anomalous IR exponents vanish since for $\delta_{g l} \geq 0$ the first equation yields directly $\delta_{g h}=0$ and the rest follows trivially.

However, so far it has not been taken into account in our scaling analysis that the DSEs have to be renormalized. As shown in [11, 13] when a propagator is divergent, it is possible to do this renormalization at $Q^{2}=0$ which cancels the tree level part identically. Thereby, the renormalization condition can act as a boundary condition for the DSEs, as observed in [23]. Since the exponent of the gluon propagator is non-negative, as shown before, this renormalization prescription is not possible there. In contrast due to this positivity the mixed loop correction in the ghost equation proves that the ghost propagator can indeed be divergent and the two exponents are then connected by $\delta_{g h}=-\delta_{g l} / 2$. Because of this connection the equation for the gluon propagator is dominated by the ghost loop, but it becomes trivial and does not determine $\delta_{g l}$. The solution depends therefore on a free parameter $\delta_{g l} \equiv 2 \kappa \geq 0, \delta_{g h}=-\kappa \leq 0$. With the expressions for the propagators this immediately gives $\delta_{3 g}=-3 \kappa$ from the ghost loop which shows that the ghost loop dominates the gluon loop also in the four gluon vertex DSE and we have $\delta_{4 g}=-4 \kappa$. Therefore, there is only a single scaling solution of this system depending on a real parameter $\kappa \geq 0$ when the relations arising from the condition of a stable skeleton expansion eq. (22) are taken into account. This is the solution obtained previously in [14 16] where it was shown that the above ghost dominance mechanism holds for arbitrary $n$-point functions. Ghost loop contributions to gluonic correlation functions and minimal mixed loop contributions to correlation functions involving ghosts dominate and all gluonic corrections are suppressed due to the scaling of the propagators despite the strongly divergent gluonic vertex functions. Here, in particular the trivial solution $\delta_{i}=0, \forall i$, obtained for a generic renormalization prescription is also contained in the above scaling solution for $\kappa=0$. The restriction to solutions of the scaling type arises here since we implicitly assumed that the integrals are always dominated by modes in the vicinity of soft external momentum scales. In the next section we will see that this is too simplified and that the result can be changed by the presence of large scales.

\section{INCLUSION OF SOFT SINGULARITIES AND MASSES}

As discussed in detail in section II, the IR counting is complicated by the possibility of kinematic divergences or dynamical mass generation. In these cases there are hard external scales present that do not tend to zero when taking the IR limit and care has to be taken to assess how a certain correlation function scales with the soft momenta. Whereas for the propagators there are unique anomalous IR exponents, in general there can be different IR exponents that describe how a correlation function scales in different kinematic sections. The uniform limit where all external scales go to zero uniformly presents the conformal case studied above. Beyond this, there are for the 3-point functions possibly distinct IR exponents when only a single momentum vanishes, as illustrated in fig. 7. According to eq. (2), we discriminate these different kinematic limits by upper indices and denote the corresponding uniform indices in the following by $\delta_{g g}^{u}$ and $\delta_{3 g}^{u}$. There are in addition the exponents for the ghost-gluon and 3-gluon vertices, when a gluon momentum vanishes, $\delta_{g g}^{g l}$ and $\delta_{3 g}^{g l}$, respectively, and for the ghost-gluon vertex, when a ghost momentum vanishes, $\delta_{g g}^{g h}$. As illustrated in fig. 8, for the 4-gluon vertex, studied in the uniform limit in [31, there can be two distinct additional kinematic exponents $\delta_{4 g}^{g l}$ when one or $\delta_{4 g}^{2 g}$ when two gluon momenta vanish. In addition there could even be IR divergences when all external momenta are large but differences of momenta become small. Because of the symmetry of the vertex this can be described by one additional IR exponent $\delta_{4 g}^{i}$ corresponding to the exchange of a soft momentum in an intermediate channel.

Although possible, it is rather cumbersome to determine the IR exponent of the 2-loop graphs appearing in some DSEs taking into account the various possible kinematic singularities. Fortunately, from what we know from the analysis in the uniform limit this should not be necessary. There the observed strong ghost dominance strongly suppressed gluonic contributions compared to the leading ghost loops. Because of the absence of primitively divergent 4-point interactions involving ghosts all of these leading contributions involve only 3-point vertices and are 1-loop graphs. Assuming that the additional kinematic singularities do not entirely change this property motivates a truncation scheme involving only dressed 3-point vertices neglecting all 2-loop graphs and those involving dressed 4-point vertices. The only graphs that involve bare 4-point vertices that are not 2-loop are those discussed in sec. III and which provided the mere constraint $\delta_{g l} \geq 0$. Therefore we will not have to discuss them here again. The truncation we study here is also similar 
to the one obtained from a 3-loop expansion of a 3PI action analyzed in 32 but with the difference that in the DSEs there is one bare vertex in every graph.

To recapitulate, the general assumptions of the IR analysis introduced in section [I] are

(i) that the local degrees of freedom are valid to describe the system,

(ii) that the IR regime of the DSE system can be analyzed using a skeleton expansion and

(iii) that the IR is described by power law scaling.

Furthermore, in this section only

(iv) we truncate the system to 1-loop graphs to simplify the analysis.

We emphasize that once solutions are found it can be explicitly verified that they present consistent IR solutions of the full DSE system. Green functions are then dominated by the leading order skeleton contributions involving primitively divergent Green functions, i.e. the assumptions (i) \& (iv) are indeed fulfilled. The assumptions (ii) \& (iii) merely limit the class of solutions that can be found with our technique.

In the case that several external scales are present the loop integral can receive relevant contributions from fluctuations in the vicinity of all these different scales. Therefore, as discussed in section II] it is necessary to decompose the momentum integral into distinct integrals which in the IR limit are entirely dominated in the respective soft or hard momentum regions. These integrals involve only one characteristic scale each and their IR scaling can then again be determined by power counting. Furthermore, we have to take into account the possibility of dynamical mass generation. Generally the presence of masses is similar to hard momentum scales in that the hard momentum region of loop integrals can be important. The extended power counting analysis that takes into account all these aspects has been presented in section II] where the possibility of dynamical mass generation was linked to the IR exponents of the propagators via the auxiliary symbols in eq. 13 for the gluons $\mu_{g l}$ and ghosts $\mu_{g h}$.

In the presence of several different scales a mere power counting of anomalous IR exponents is not sufficient anymore but the canonical scaling of the integrals, propagators and vertices has to be considered. In particular it is possible that tensor structures of a vertex involve hard momenta and do not scale with the soft momentum. Correspondingly, it is also necessary to discriminate between bare and dressed vertices in this context. Therefore we will first assess the canonical scaling of the appearing vertices in detail. Let us start with the bare ghost-gluon vertex which in our conventions depends only on the outgoing ghost momentum. If this momentum is soft the canonical scaling has to be taken into account independent of the size of the other momenta and vice versa. In addition, when the vertex is connected to an internal gluon propagator the tensor in the direction of the gluon momentum is canceled due to the transversality of the gluon propagator in Landau gauge

$$
q^{\mu} D_{\mu \nu}(p)=\left(q-\frac{p \cdot q}{p^{2}} p\right)^{\mu} D_{\mu \nu}(p) \equiv q_{\perp}^{\mu} D_{\mu \nu}(p) .
$$

The dressed ghost-gluon vertex has two independent tensor structures that can be chosen as arbitrary linear combinations of the 3 external momenta. If only one external momentum is soft there is a tensor structure that depends on hard momenta which will dominate as long as there are no cancelations. Therefore a dressed vertex has generically no canonical scaling whenever there are hard scales involved. However, because of the above transversality this can be changed when there is only one hard external scale - as is the case in propagator integrals - but not if there are two independent ones.

The 3-gluon vertex has many tensor structures but it turns out to be sufficient to analyze the tree level tensor

$$
\left(\Gamma_{0}\right)_{\mu \nu \rho}^{a b c}(p, q, r)=-i g f^{a b c}\left((p-q)_{\rho} \delta_{\mu \nu}+(q-r)_{\mu} \delta_{\nu \rho}+(r-p)_{\nu} \delta_{\rho \mu}\right)
$$

When only one of the momenta is soft the tensor is in general of the order of the hard momenta that dominate soft contributions. Other possible tensor structures that depend only on soft momenta are likewise subleading compared to the tree level tensor and the canonical scaling is again not present. Gluon transversality cannot directly change this here since the above tensor structure involves the metric tensor which couples the two attached gluon propagators in a loop directly. However, there are cases where the Bose symmetry of the vertex can lead to additional cancelations as discussed below.

Let us now apply this to the individual diagrams to obtain the corresponding equations for the IR exponents. The decomposition of the full ghost DSE reads 


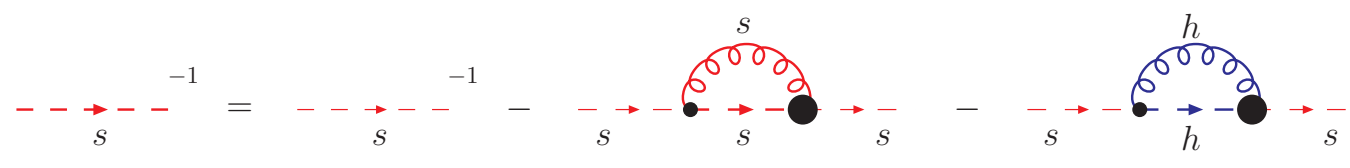

Since it depends only on a single momentum the dominant loop momenta that contribute in the loop graph are normally of the order of the soft external momentum scale $k \sim p$ but when masses are generated dynamically also much larger momenta $k \sim \Lambda_{Q C D} \gg p$ can contribute and could in principle be relevant due to the positive mass dimension of the graph. In particular, because of the possibility of kinematic singularities of the vertices one obtains a nontrivial contribution from large loop momenta that involve the IR exponent of the ghost-gluon vertex in the limit that the ghost leg becomes soft. Although the integration measure for this contribution from hard momenta does not scale with the soft external momentum, it is suppressed due to cancelations owing to the gluon transversality eq. 25) which introduce a canonical scaling part for the ghost-gluon vertices. This immediately rules out the possibility of an IR constant ghost propagator since the leading contributions from finite modes that could produce such an IR ghost mass $m_{g h}(p) \gg p$ are canceled in the ghost integral and only subleading contributions remain. Because of the absence of such a mass $\mu_{g h} \equiv 1$ and there is the possibility of an additional suppression of the propagator eq. 12 given by $\mu_{g l}$ in case the gluon propagator is likewise massless. The corresponding equation for the anomalous dimension of the ghost reads therefore

$$
-\delta_{g h}+1=\min \left(1 ; \delta_{g g}^{u}+\delta_{g h}+\delta_{g l}+1, \delta_{g g}^{g h}+1+\mu_{g l}\right),
$$

where here and in the following we separate terms arising from different graphs by semicolons and those arising from different kinematic regions of the same graph by commas.

The gluon equation truncated to IR leading 1-loop terms is given by

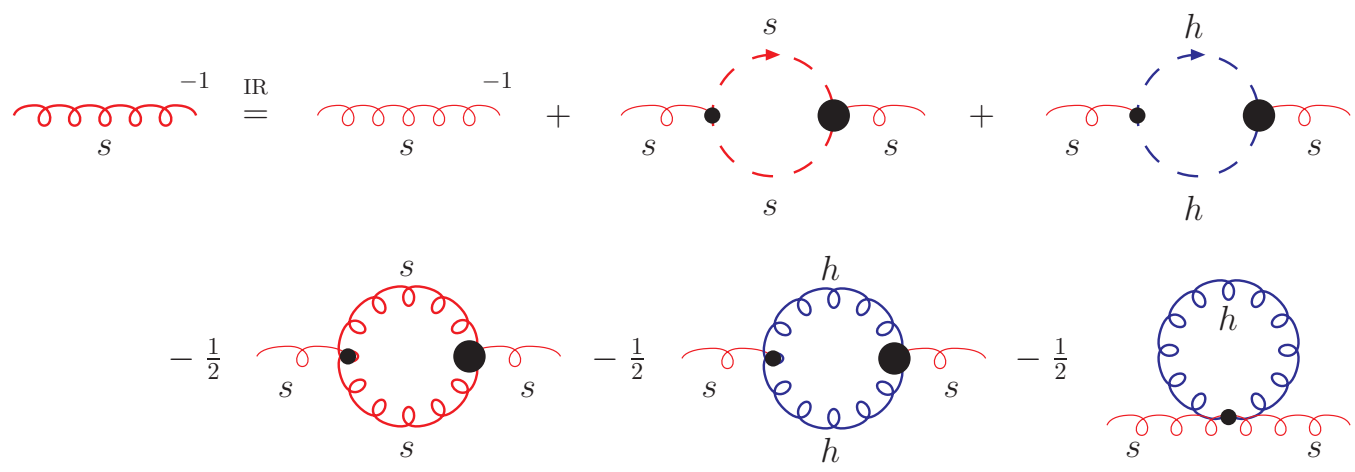

As in the ghost case there can be contributions from the hard part of the loop integral. In particular, in this case there is no suppression due to transversality as in the loop correction of the ghost propagator and therefore an IR gluon mass is not ruled out. The absence of an IR ghost mass implies that according to eq. 12 the contribution from the hard region of the ghost loop is suppressed by $p^{2} / k^{2}$ so that this correction cannot induce an IR gluon mass. This is different for the gluonic corrections that can contribute once a gluon mass has been dynamically generated but are likewise suppressed otherwise. Since the external scale does not enter the tadpole correction at all, it is not necessary to distinguish between different kinematic regions in this case and as long as the there is no scale induced this contribution is canceled identically. Correspondingly, the gluon equation reads

$$
-\delta_{g l}+1=\min \left(1 ; \delta_{g g}^{u}+2 \delta_{g h}+1, \delta_{g g}^{g l}+1 ; \delta_{3 g}^{u}+2 \delta_{g l}+1, \delta_{3 g}^{g l}+\mu_{g l} ; \mu_{g l}\right),
$$

The leading contributions in the ghost-gluon DSE are given by the two triangle graphs that are analogous to the Abelian and non-Abelian diagrams in the quark-gluon vertex. As discussed in section II] when scales of different order of magnitude are involved, the decomposition of the loop integrals yields in addition to a hard contribution generally two contributions from inequivalent kinematic regions of the loop integral shown in fig. 10 where different internal momenta become soft. These correspond to inequivalent ways to route the large momentum through the loop.

E.g. for hard and soft external momenta $p$ and $q$, respectively, and an assignment of the loop momentum $k$ such that the first IR relevant contribution in fig. 10 arises from soft loop momenta $k \sim q$, the second one arises from hard 


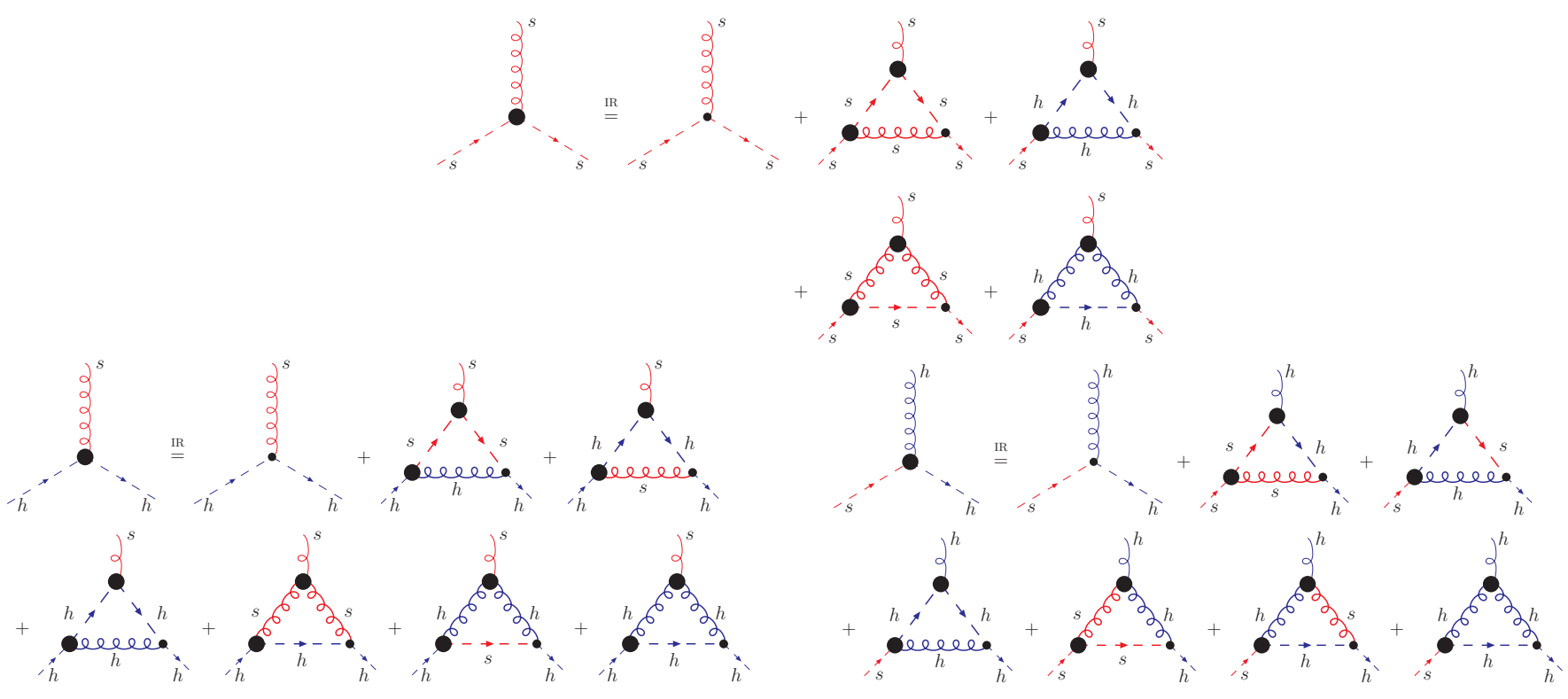

Figure 13: The IR leading part of the decomposed equations for the ghost-gluon vertex in the uniform (top), soft-gluon (bottom left) and soft-ghost limit (bottom right).

loop momenta in a correspondingly narrow momentum interval $k-p \sim q \ll p \sim k$. When assessing the counting of the vertex, each of these kinematic contributions of the initial loop integral could dominate and has to be taken into account separately. Using the graphical representation depicted in figs. 10 and 11 the ghost-gluon DSE in the different kinematic limits is explicitly represented in fig. 13 . We should stress that there is no double counting involved here, since the figure only visualizes the different distinct IR-sensitive regions of the loop integral that arose from the exact decomposition discussed in section $\Pi$ and appendix $B$.

From the decomposed DSE we obtain the IR counting of the individual contributions to the ghost-gluon vertex in the uniform limit

$$
\delta_{g g}^{u}+\frac{1}{2}=\min \left(\frac{1}{2} ; 2 \delta_{g g}^{u}+2 \delta_{g h}+\delta_{g l}+\frac{1}{2}, \delta_{g g}^{g l}+\delta_{g g}^{g h}+1+\mu_{g l} ; \delta_{3 g}^{u}+\delta_{g g}^{u}+\delta_{g h}+2 \delta_{g l}+\frac{1}{2}, \delta_{3 g}^{g l}+\delta_{g g}^{g h}+1+\mu_{g l}\right) .
$$

Here in the minimum function the first element is the bare vertex, the next two arise from the regions of soft and hard loop momenta of the "Abelian" graph whereas the final two are the contributions from the corresponding regions of the "non-Abelian" graph. In the contributions from hard momenta the 1 arises again from cancelations due to the gluon transversality. In the limit that only the gluon becomes soft we find

$$
\delta_{g g}^{g l}=\min \left(0 ; \delta_{g g}^{u}+\delta_{g g}^{g h}+2 \delta_{g h}+\frac{3}{2}, 2 \delta_{g g}^{g l}+\delta_{g l}+1, \delta_{g g}^{g l} ; \delta_{3 g}^{u}+\delta_{g g}^{g l}+2 \delta_{g l}+\frac{1}{2}, \delta_{3 g}^{g l}+\delta_{g g}^{g h}+\delta_{g h}+2, \delta_{3 g}^{g l}+\frac{1}{2}\right) .
$$

The additional suppression of the last term from the hard momentum region of the "non-Abelian" graph arises from the combination of gluon transversality and the Bose symmetry of the 3-gluon vertex. Choosing as the basis for the dressed ghost-gluon vertex the outgoing ghost- and the gluon-momentum, $\Gamma_{g g}=A p_{g h}^{\text {out }}+B p_{g l}$, with corresponding dressing functions $A$ and $B$, the contraction of the dressed and bare ghost-gluon vertex with the corresponding gluon propagators in the loop gives, respectively,

$$
\begin{aligned}
\left(A \cdot(k+h+s)_{\gamma}+B \cdot(k+s)_{\gamma}\right)\left(\delta_{\gamma \alpha}-\frac{(k+s)_{\gamma}(k+s)_{\alpha}}{(k+s)^{2}}\right) & \approx A \cdot\left(h_{\alpha}-\frac{k \cdot h}{k^{2}} k_{\alpha}\right)+O(s), \\
(h+s)_{\delta}\left(\delta_{\delta \beta}-\frac{k_{\delta} k_{\beta}}{k^{2}}\right) & \approx h_{\beta}-\frac{k \cdot h}{k^{2}} k_{\beta}+O(s),
\end{aligned}
$$

where $h$ is the incoming hard external ghost momentum, $s$ the soft external gluon momentum and $k$ the loop momentum. Because of the antisymmetry of the color part, the Bose symmetry of the dressed 3-gluon vertex implies that it 

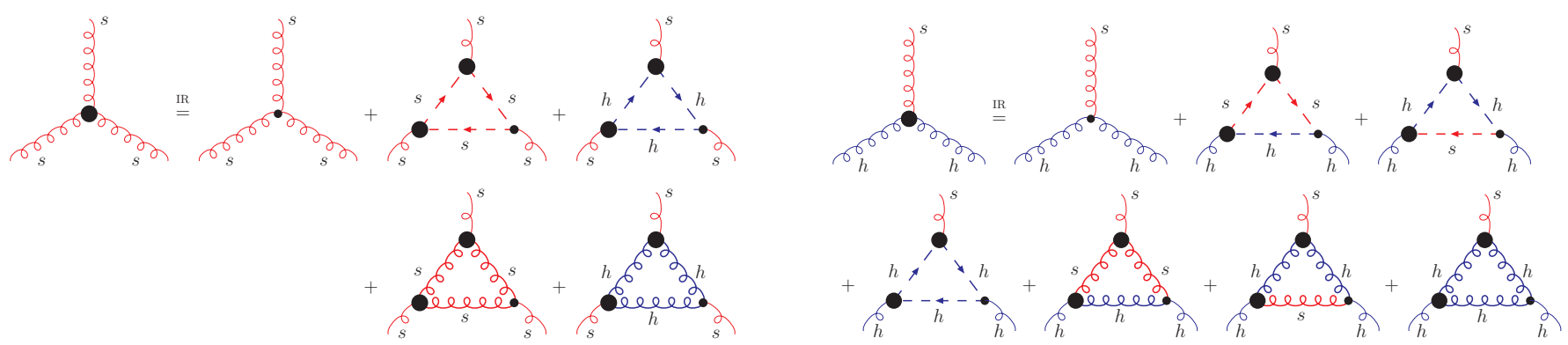

Figure 14: The IR leading part of the decomposed equations for the 3-gluon vertex in the uniform (left) and soft-gluon limit (right).

is antisymmetric with respect to simultaneous commutation of momenta and Lorentz indices. The appearance of two identical momenta up to $O(s)$ corrections shows then that the leading term vanishes and the loop scales actually as $O(s)$. The corresponding equation in the soft ghost limit follows similarly from the power counting analysis

$$
\delta_{g g}^{g h}=\min \left(0 ; \delta_{g g}^{u}+\delta_{g g}^{g h}+\delta_{g h}+\delta_{g l}+\frac{1}{2}, 2 \delta_{g g}^{g h}+\delta_{g h}+2, \delta_{g g}^{g h} ; \delta_{g g}^{u}+\delta_{3 g}^{g l}+\delta_{g h}+\delta_{g l}+1, \delta_{3 g}^{g l}+\delta_{g g}^{g h}+\delta_{g l}+\frac{3}{2}, \delta_{g g}^{g h}\right)
$$

Finally in the considered truncation there is the equation for the 3-gluon vertex where the contributions are given by the ghost and gluon triangles. The equations for the two different kinematic limits are shown in fig. 14. In the uniform limit the power counting yields the equation

$$
\delta_{3 g}^{u}+\frac{1}{2}=\min \left(\frac{1}{2} ; 2 \delta_{g g}^{u}+3 \delta_{g h}+\frac{1}{2}, 2 \delta_{g g}^{g l}+1 ; 2 \delta_{3 g}^{u}+3 \delta_{g l}+\frac{1}{2}, 2 \delta_{3 g}^{g l}+\frac{1}{2}+\mu_{g l}\right),
$$

where the $1 / 2$ in the contribution from hard modes of the gluon triangle arises since the leading term depending only on the loop momentum is odd and vanishes in the symmetric integration. The soft-gluon limit requires some more care. Here, the IR exponent of the ghost triangle seems to depend on different factors like the loop routing, the definition of the bare ghost vertex or which of the three vertices is taken bare in the DSE. All these factors seemingly determine whether the appearing bare ghost-gluon vertex scales canonically. To see that this is actually the case independent of all these conventions, it is important to remember that the 3 -gluon vertex is totally symmetric. This means that all three ways of assigning the external momenta and indices to the external legs of the graph yield the same result. With the standard convention that the bare vertex is proportional to the outgoing ghost momentum, this momentum is soft for at least one of the three configurations. Since the IR exponent obtained by the power counting analysis can overestimate the degree of divergence of a given graph when there are cancelations but it cannot underestimate it, the bare ghost-gluon vertex features indeed a canonical scaling. Correspondingly one obtains here

$$
\delta_{3 g}^{g l}=\min \left(0 ; \delta_{g g}^{u}+\delta_{g g}^{g h}+2 \delta_{g h}+1, \delta_{g g}^{g l}+\delta_{g g}^{g h}+\delta_{g h}+\frac{3}{2}, \delta_{g g}^{g l} ; \delta_{3 g}^{u}+\delta_{3 g}^{g l}+2 \delta_{g l}+\frac{1}{2}, 2 \delta_{3 g}^{g l}+\delta_{g l}+1, \delta_{3 g}^{g l}\right) .
$$

The fourth element in the minimum function gives a constraint on the two vertices in the soft gluon limit $\delta_{3 g}^{g l} \leq \delta_{g g}^{g l}$. It is straightforward to see that the reduced system of the three equations for the soft divergences alone is consistent with the uniform solution discussed in the last section if the corresponding IR exponents are taken as given. Though, here we want to discuss the general case where the additional kinematic divergences couple back and could thereby change the uniform solution. To this end we study the full, coupled system of seven equations obtained by the consideration of possible kinematic divergences.

We want to stress that, analogous to the case discussed in detail in section III the IR exponents on the left-hand side in the vertex eqs. (29), (30), (32) and (34) appear linearly also on the right-hand side. The corresponding inequalities, e.g. $\delta_{g g}^{u}+1 / 2 \leq \delta_{g g}^{u}+\delta_{3 g}^{u}+\delta_{g h}+2 \delta_{g l}+1 / 2$ from eq. (29), can be fulfilled independently of the values of the IR exponents on the left-hand side, since they drop out. Yet, they provide constraints on the other IR exponents. From a purely mathematical point of view these DSEs could be trivially fulfilled for a whole range of values for the corresponding IR exponent if these linear terms would dominate. Yet, such linear terms provide no non-linear feedback and cannot alter the degree of divergence compared to the tree level term in the DSE that is not IR enhanced. Correspondingly 
from a physical point of view these terms are irrelevant for the IR solution.

As in the uniform case the system of IR equations is again constrained by necessary conditions for a stable skeleton expansion. In addition to those in the uniform limit eqs. (22) there are corresponding constraints from the graphs in fig. 12 when the loop momentum is in the IR regime but one of the two connected propagators has a hard momentum

$$
\begin{aligned}
\delta_{g g}^{u}+\delta_{g g}^{g l}+\delta_{g h}+\delta_{g l}+\frac{1}{2} & \geq 0, \\
\delta_{3 g}^{u}+\delta_{g g}^{g l}+2 \delta_{g l}+\frac{1}{2} & \geq 0, \\
\delta_{g g}^{u}+\delta_{3 g}^{g l}+\delta_{g h}+\delta_{g l}+\frac{1}{2} & \geq 0, \\
\delta_{3 g}^{u}+\delta_{3 g}^{g l}+2 \delta_{g l}+\frac{1}{2} & \geq 0,
\end{aligned}
$$

or when the momenta of both connected propagators are hard

$$
\begin{aligned}
2 \delta_{g g}^{g l}+\delta_{g l}+1 & \geq 0, \\
\delta_{3 g}^{g l}+\delta_{g g}^{g l}+\delta_{g l}+1 & \geq 0, \\
2 \delta_{3 g}^{g l}+\delta_{g l}+1 & \geq 0 .
\end{aligned}
$$

In principle there may also be constraints from the hard part of the added loop, but in this case the extension can change the counting far away from the insertion and therefore they do not take a simple form. Using the constraints eqs. 22, 35 and 36 as well as $\delta_{3 g}^{g l} \leq \delta_{g g}^{g l}$, the system of inequalities, eqs. 27)-(34), reduces to:

$$
\begin{aligned}
-\delta_{g h}+1 & =\min \left(1, \delta_{g g}^{u}+\delta_{g h}+\delta_{g l}+1, \delta_{g g}^{g h}+1+\mu_{g l}\right), \\
-\delta_{g l}+1 & =\min \left(1, \delta_{g g}^{u}+2 \delta_{g h}+1, \delta_{3 g}^{u}+2 \delta_{g l}+1, \delta_{3 g}^{g l}+\mu_{g l}\right), \\
\delta_{g g}^{u}+\frac{1}{2} & =\min \left(\frac{1}{2}, \delta_{3 g}^{g l}+\delta_{g g}^{g h}+1+\mu_{g l}\right), \\
\delta_{3 g}^{u}+\frac{1}{2} & =\min \left(\frac{1}{2}, 2 \delta_{g g}^{u}+3 \delta_{g h}+\frac{1}{2}, 2 \delta_{3 g}^{g l}+\frac{1}{2}+\mu_{g l}\right), \\
\delta_{g g}^{g h} & =\min \left(0,2 \delta_{g g}^{g h}+\delta_{g h}+2\right), \\
\delta_{g g}^{g l} & =\min \left(0, \delta_{g g}^{u}+\delta_{g g}^{g h}+2 \delta_{g h}+\frac{3}{2}, \delta_{3 g}^{g l}+\delta_{g g}^{g h}+\delta_{g h}+2, \delta_{3 g}^{g l}+\frac{1}{2}\right), \\
\delta_{3 g}^{g l} & =\min \left(0, \delta_{g g}^{u}+\delta_{g g}^{g h}+2 \delta_{g h}+1, \delta_{g g}^{g l}+\delta_{g g}^{g h}+\delta_{g h}+\frac{3}{2}, \delta_{g g}^{g l}\right) .
\end{aligned}
$$

In the uniform case the non-renormalization of the ghost-gluon vertex was the cornerstone that allowed one to solve the corresponding system. We therefore start with this vertex in the soft ghost limit described by the equation for $\delta_{g g}^{g h}$. Let us assume for the moment that the vertex is singular so that the second term dominates. In this case we would have $\delta_{g g}^{g h}=-\delta_{g h}-2$, but this would yield a direct contradiction via the last term of the ghost propagator equation. Therefore we have instead $\delta_{g g}^{g h}=0$ and obtain additionally the weak constraint $\delta_{g h} \geq-2$. In contrast to the conformal case the remaining system allows two qualitatively different solutions:

Decoupling solution: With any renormalization prescription that does not change the above system for the IR exponents, as in the conformal case discussed above the equation for the ghost propagator as it stands has only the trivial solution $\delta_{g h}=0$. To find the solution of the residual system in the present case, we insert the remaining possible solutions for the 3-gluon vertex in the soft gluon limit into the other equations which yields

$$
-\delta_{g l}+1=\min \left(1, \mu_{g l}\right), \quad \delta_{g g}^{u}=\delta_{g g}^{g l}=\delta_{3 g}^{u}=\delta_{3 g}^{g l}=0 .
$$

In addition to the trivial fixed point, there is here also the possibility of a massive gluon $\delta_{g l}=1$. Therefore, in contrast to the conformal analysis where it was assumed that all integrals are dominated by scales of the order of the external 
momenta, hard modes of the order of the induced scale $\Lambda_{Q C D}$ can dominate the loop integrals in the equation for the gluon propagator and induce an IR gluon mass. This alternative scenario is reminiscent of the early work 24 and has recently also been suggested in [21-23] on the level of the propagator equations, whereas the present analysis shows that it is also consistent with the vertex equations. This solution is also found if the ghost propagator is renormalized to a finite value at vanishing momentum [23].

Scaling solution: Alternatively, it is again possible to use the renormalization introduced in [11, 13] as discussed in the last section which removes the tree-level term in the ghost equation. Because of the scale independence of the ghost-gluon vertex in the limit that a ghost momentum vanishes the last term in the ghost equation is either directly subleading in case the gluon propagator remains massless or can otherwise be removed in the renormalization process together with the tree-level term. Thereby the ghost equation becomes unique

$$
\delta_{g h}=-\frac{1}{2}\left(\delta_{g l}+\delta_{g g}^{u}\right)
$$

and inserting this expression for the ghost exponent, the gluon equation becomes trivially fulfilled

$$
-\delta_{g l}+1=\min \left(1,-\delta_{g l}+1, \delta_{3 g}^{u}+2 \delta_{g l}+1, \delta_{3 g}^{g l}+\mu_{g l}\right),
$$

which shows that the ghost-loop has to be the IR leading contribution. It does not provide any constraint on $\delta_{g l}$, leaving the free parameter $\kappa \equiv \delta_{g l} / 2 \geq 0$ in the solution as is known already from the uniform case. We note that the triviality of this equation is qualitatively different from what we found in the case of the equations for the kinematic divergences above, since the equation is non-linear and can therefore indeed self-consistently generate a non-trivial solution. The equation provides two additional weak constraints on the vertices $\delta_{3 g}^{u} \geq-6 \kappa, \delta_{3 g}^{g l} \geq 1-2 \kappa-\mu_{g l}$. Inserting the above expression for the ghost exponent in the first of the constraints from the skeleton expansion in the uniform limit eqs. 222 yields $\delta_{g g}^{u} \geq 0$ which makes the corresponding equation for the exponent of the uniform ghost-gluon vertex unique, so that $\delta_{g g}^{u}=0$. The residual system reads now

$$
\begin{aligned}
\delta_{3 g}^{u}+\frac{1}{2} & =\min \left(-3 \kappa+\frac{1}{2}, 2 \delta_{3 g}^{g l}+\frac{1}{2}+\mu_{g l}\right), \\
\delta_{g g}^{g l} & =\min \left(0,-2 \kappa+\frac{3}{2}, \delta_{3 g}^{g l}-\kappa+2, \delta_{3 g}^{g l}+\frac{1}{2}\right), \\
\delta_{3 g}^{g l} & =\min \left(0,-2 \kappa+1, \delta_{g g}^{g l}-\kappa+\frac{3}{2}, \delta_{g g}^{g l}\right) .
\end{aligned}
$$

Using the constraint $\delta_{3 g}^{g l} \leq 1-2 \kappa$ from the last of these equations in the third of eqs. 35 yields the constraint $\kappa<3 / 2$, so that a solution can only exist in a bounded region of the IR scaling parameter $\kappa$. The system can then be solved by inserting the possible solutions of the ghost-gluon equation in the soft-gluon limit in the equation for the 3 -gluon vertex in the same limit. Under consideration of this bound the equation reduces to $\delta_{3 g}^{g l}=\min (0,-2 \kappa+1)$. Correspondingly, the scaling of the 3-gluon vertex in the soft-gluon limit depends on the value of the IR parameter $\kappa$. For $\kappa>1 / 2$ it is singular $\delta_{3 g}^{g l}=1-2 \kappa$ whereas for $\kappa \leq 1 / 2$ it is not $\delta_{3 g}^{g l}=0$. Using these results the remaining equation becomes trivial and the scaling solution of the system is obtained. Similar to the 3 -gluon vertex the exponent in the soft-gluon limit of the ghost-gluon vertex $\min \left(0, \frac{3}{2}-2 \kappa\right)$ also depends on $\kappa$, but here a divergence would arise only for $\kappa \geq 3 / 4$. Finally, the scaling of the 4-gluon vertex in the uniform limit that satisfies the DSE system is obtained from the corresponding DSE fig. 6 as before and yields the known result $\delta_{4 g}^{u}=-4 \kappa$ whereas the determination of the corresponding kinematic divergences requires a more detailed study. In summary, the IR fixed points for the two qualitative distinct solutions are given by Table I

These solutions fulfill all constraints that appeared in the course of the evaluation and present therefore refined IR fixed points of Landau-gauge Yang-Mills theory. Several remarks are in order at this point:

- In this work we restricted our analysis to the IR behavior of Yang-Mills theory. The physical relevance of such a study stems certainly from the fact that Yang-Mills theory presents the gauge sector of QCD and might therefore provide important insight into qualitative properties of the strong interaction. Whereas an independent study of the gauge dynamics is by definition sufficient in the quenched limit [6], in the case of dynamical QCD it is not a priori clear that the quark dynamics does not affect the IR fixed point structure of gauge Green functions obtained here. Yet, a corresponding recent IR analysis of QCD [7, based on the general methods developed in this work, shows that the gauge sector is totally unaltered by the quark dynamics and thereby strongly supports the relevance of the results presented here and in other studies of Yang-Mills theory. 


\begin{tabular}{|c|c|c|c|c|c|c|c|c|}
\hline & $\delta_{g h}$ & $\delta_{g l}$ & $\delta_{g g}^{u}$ & $\delta_{3 g}^{u}$ & $\delta_{4 g}^{u}$ & $\delta_{g g}^{g h}$ & $\delta_{g g}^{g l}$ & $\delta_{3 g}^{g l}$ \\
\hline scaling & $-\kappa$ & $2 \kappa$ & 0 & $-3 \kappa$ & $-4 \kappa$ & 0 & $\min \left(0, \frac{3}{2}-2 \kappa\right)$ & $\min (0,1-2 \kappa)$ \\
\hline decoupling & 0 & 1 & 0 & 0 & 0 & 0 & 0 & 0 \\
\hline
\end{tabular}

Table I: The IR exponents for the leading Green functions of the IR fixed points of Landau gauge Yang-Mills theory within the two possible IR scenarios. The scaling analysis restricts the parameter to be only positive $\kappa \geq 0$, and yields rather weak upper bounds. (Note that these bounds on $\kappa$ are weaker than the one that had been erroneously given in a preprint version of this article. Stronger bounds $0.5 \leq \kappa \leq 1$ are, however, supported by analyses of the actual loop integrals [12].)

- In contrast to the case of the conformal analysis discussed in the previous section, where the chosen boundary condition merely excluded other solutions, the decoupling solution obtained for a generic condition is qualitatively different from the scaling solution obtained for a choice that had an unbroken global BRS charge [23. Whereas in the scaling solution the ghosts are strongly IR enhanced resulting in divergent gluonic vertices, in the decoupling solution neither the ghosts nor the vertices are anomalously enhanced. This strongly suppresses any IR dynamics mediated by the gluons in the ratio $\rho \equiv p^{2} / m_{g l}^{2}$ where $m_{g l}$ is the finite IR limit of the gluon polarization. Thereby it is easy to see that in both cases the leading contribution to a Green function is given by the ghost dynamics. In the decoupling case the IR exponents in Table 1 show directly that the leading term in the skeleton expansion of a general vertex with $n$ ghost-pairs is anomalously suppressed by $\rho^{n}$ whereas purely gluonic Green functions scale canonically. In addition to the given decoupling solution there might be further IR fixed points where even the vertices decouple and become IR constant [33].

- Recent lattice simulations on large lattices [3] show a gluon propagator that does not feature a decrease in the IR and a ghost propagator that is basically not IR enhanced and thereby favor the decoupling scenario. Moreover, it has been argued that this is probably neither a finite volume [34] nor a statistical effect [35]. However, there seem to be issues with Gribov copies [36, 37] and discretization effects 38, in these analyses. Such effects could shadow a potential scaling behavior in the deep IR, so that the scaling scenario cannot be excluded with the present data. Another view is presented in [39], where it is suggested that the different boundary conditions 23] correspond to distinct residual gauge fixing conditions. In principle Gribov copies could be an issue in the DSE system as well and the constraint to the fundamental modular region that is free of Gribov copies could change these equations and their solution structure. However, it has recently been shown explicitly [40 that at least the restriction to the first Gribov region [5] using the Gribov-Zwanziger action [41] does not affect the IR fixed point structure of the scaling solution.

- The IR exponents given in Table I determine only the anomalous scaling laws for the most singular tensor parts. The scaling of the full Green functions involves also the canonical scaling dimension incorporated in the tensors. In particular it is possible that some dressing functions are more IR singular than the leading dressing function, but their contribution to the vertex is nevertheless subleading since it is additionally suppressed by the canonical scaling of their tensor. As we show in [20] this is indeed the case for the ghost-gluon vertex which features more structure than the above result suggests. Instead, a soft-gluon singularity appears in the form factor of the longitudinal tensor that is additionally suppressed by the gluon momentum in the tensor and actually IR vanishing, whereas the tree-level tensor is entirely IR finite and presents the IR leading structure. In order to reveal such subtleties in our power counting analysis we would have had to include different anomalous dimensions for the different tensor structures. Since we present an explicit analytic solution for the IR limit of the 3-point vertices in [20] we refrained here from such complications. In [20] we show explicitly that the transverse part of the 3-gluon vertex does not have leading tensors. The analytic result obtained there supports this observation. It also explains why the kinematic singularity for the three-point vertices obtained in the second work of ref. [15], where only the transverse part was considered, is lower as the one given in tab. I]

- The kinematic singularities do not alter but merely extend the previously know uniform scaling fixed point. Yet, the soft singularities restrict the range of possible $\kappa$-values from the mere positivity requirement in the conformal case to the bounded interval $0 \leq \kappa \leq 3 / 2$. The trivial solution is included in the scaling solution for $\kappa=0$. It is (up to logarithmic corrections) realized in the UV regime of the theory characterized by asymptotic freedom and it is clear from the perturbative $\beta$-functions that this solution can hardly be a stable IR fixed point, too. The best currently known value for the IR scaling parameter is $\kappa \approx 0.5953$ [12, 13] obtained from an analytic IR solution of the integrals in the DSEs for the propagators.

- Interestingly below $\kappa=1 / 2$ the kinematic singularities entirely disappear. The latter value is a special case since the gluons show effectively a massive behavior but the ghost is in contrast to the corresponding decoupling solution still strongly divergent. For $\kappa>1 / 2$ the gluon propagator should vanish with the small exponent $2 \kappa-1$ 
which is precisely the negative of the exponent for the mild kinematic singularities of the 3-gluon vertex found here. In four dimensions such a small exponent naturally poses a huge numerical challenge and is not observed in current studies [3], but in lower dimensions a corresponding decrease has been clearly confirmed [42. Similarly, it is not surprising that the predicted kinematic singularities have not been seen so far in present vertex studies [43] which are numerically even much more challenging than those for the propagators. In [20] we proposed a suitable tensor contraction for the three-gluon vertex to investigate the existence of kinematic singularities on the lattice.

- It is crucial that the ghost-gluon vertex is finite when only a ghost momentum vanishes. This result follows immediately from the corresponding "un-decomposed" DSE which contains only a single loop graph involving the connected (instead of 1PI) ghost-gluon scattering kernel 14. By transversality this graph is directly proportional to the external momentum and leaves only the tree level part in the IR limit in accordance with the nonrenormalization of this vertex. We point out that there is, however, no corresponding argument when the gluon momentum vanishes.

- The obtained divergence when only a single gluon momentum vanishes naively seems to be problematic for several reasons: First of all it seems to induce an even stronger singularity in the ghost-gluon vertex in the uniform limit from hard loop momenta. As pointed out above though in this case the transversality in Landau gauge prevents this and instead makes this contribution strongly subleading. This is also in accordance with the two different versions for the ghost-gluon vertex. Since a dressed 3-gluon vertex is present only in the first one, the two versions would be inconsistent if the kinematic-divergence of the 3-gluon vertex would alter the degree of divergence of the full vertex. Secondly, naively there seems to be a huge problem with the soft gluon singularity in the 3-gluon vertex. First of all it arises directly from the ghost loop integral with dressed propagators. But once induced, it seems to arise in addition also in dressed vertices whenever the external momentum becomes soft and totally independent of the loop integral. This would enhance the divergence in each iteration and make it more and more divergent. As seen explicitly in the above analysis the reason why this is not the case is that the hard region is additionally suppressed and that both of these different singularities arise from distinct regions of the loop integration and thereby cannot amplify each other.

- As found from the analysis above, all Green functions in the scaling solution include a graph that does not involve singular vertices. In particular, the IR dominant ghost loop correction to the 3-gluon vertex induces the soft-gluon divergence entirely due to the enhancement of the ghost propagator so that the appearance of kinematic divergences is a direct consequence of the ghost dominance of the uniform solution. This allows one to capture the qualitative IR behavior of the vertices in a semi-perturbative scheme that involves dressed propagators but employs bare vertices. This approximation is used in a companion article and allows a complete analytic solution in terms of hypergeometric functions [20. Although the analysis of the kinematic divergences of the 4-gluon vertex is more complicated and requires a detailed analysis, we note here that the semi-perturbative contribution from the ghost loop yields $\delta_{4 g}^{g l}=1-2 \kappa$ and $\delta_{4 g}^{2 g}=1 / 2-3 \kappa$ which due to the ghost dominance is expected to be the leading contribution. Incidentally this is exactly the scaling of the 3-gluon vertex in the soft-gluon and uniform limit whereas the uniform limit of the 4-gluon vertex $\delta_{4 g}^{u}=-4 \kappa$ is more divergent by yet another $-1 / 2-\kappa$. These results suggest the conjecture that the full scaling of a gluonic correlation function with $n_{s}$ independent soft external momenta should be $\Gamma_{n_{s}} \sim\left(p^{2}\right)^{2-\left(n_{s}+1\right)(1 / 2+\kappa)}$.

- Inserting the results for the scaling solution in Table $\mathbb{1}$ in the constraints for the skeleton expansion involving hard momenta we find that the extensions count as $3 / 2-\kappa$ and $3-2 \kappa$ when there are one respectively two hard propagators in the extended graph. With the above limits for $\kappa$ this shows that these extensions are strongly suppressed and correspondingly such extensions do not have to be taken into account in the skeleton expansion. In contrast, inserting the results in the constraints for the skeleton expansion in the uniform limit eqs. 22, it is clear that all of them are saturated and correspondingly all orders in the expansion scale equally [14]. Note at this point, that we did not assume by our constraints eqs. 22 that the skeleton expansion strictly converges, but only that it is not explicitly divergent. In general, the skeleton expansion could be an asymptotic series as suggested by the IR scaling. Therefore, the whole tower of such graphs had to be resummed which could in principle change the IR scaling. For instance it is well known from standard resummed perturbation theory that the resummation of perturbative logarithms yields a power law scaling with an anomalous exponent. However, even such logarithmic divergences can be invariant under resummation, as e.g. found for the non-Fermi liquid corrections in dense QCD [44. In the current case a resummation seems to be impossible in full generality, anyhow, but the decisive difference is that the graphs that are resummed already feature power law scaling and therefore we expect that they are indeed invariant under resummation. Furthermore, it has been shown in [15, 27, 45] that the uniform IR solution can be obtained independently of the skeleton expansion and the same will be shown for the more general case as discussed in a forthcoming publication [7, 46]. 
- More generally the validity of the skeleton expansion is closely linked to the existence of any finite truncation of the DSEs and eventually to the concept of locality. To see this, note that an explicitly divergent skeleton expansion suggests that there is no finite approximation to describe higher order Green functions by lower ones or in particular only in terms of the primitively divergent correlators. This would mean that the higher Green functions include important physics that is not yet included in the lower Green functions and that is required to properly describe the system. In particular these higher Green functions in turn significantly influence the solution of the leading Green functions within an explicit analysis of the dynamics in this case. Any truncation of the system with a local effective action that includes only a finite number of terms would thereby miss the main physics and instead a non-local effective action with an infinite number of terms in the local fields is necessary. Such a non-local situation means that the description in terms of local degrees of freedom breaks down and the system can probably be more conveniently described by non-local degrees of freedom with a finite number of terms in the action. Now one could argue that, since one is only interested in the behavior of the leading Green functions, as far as the appearing higher order Green functions in the corresponding DSEs are known (e.g. the 5- \& 6-point functions in Yang-Mills theory) the system for the primitively divergent Green functions may be solved independent of these problems. The important point to realize is that in such a case there is no way to obtain them, neither in the context of functional techniques like DSEs nor in any other scheme like lattice gauge theory since even there the number of lattice points corresponds to the highest Green function that can be realized and that could contribute to the dynamics of the system in this truncation. Correspondingly, in contrast to the usual situation where one expects that the precise form of the unknown higher Green functions becomes irrelevant at sufficiently high order and thereby a sensible ansatz should be given by general arguments like symmetry restrictions, this is intrinsically not fulfilled in case of an explicitly divergent skeleton expansion. Finding a reasonable ansatz for these higher order Green functions is thereby equivalent to guessing the correct solution for the primitively divergent Green functions in the first place. Finally, even if we would simply by chance guess the precise solution for the required higher Green functions to solve the system for the lowest correlators like the propagators, the above line of reasoning shows that these local Green functions would have nothing to do with the actual physics of the system since these degrees of freedom should not be suitable to describe the system in case of a divergent skeleton expansion. From this point of view we regard the existence of a stable skeleton expansion as a rather physical requirement for any analysis in terms of underlying local degrees of freedom.

\section{CONCLUSIONS}

We have presented a power counting formalism for the analysis of the non-perturbative IR behavior of field theories in the general case when finite scales are present. This allows the inclusion of kinematic divergences and dynamical mass generation in the power counting analysis. In this framework we studied the IR regime of Landau gauge YangMills theory in more detail and found that the fixed point structure is more diverse than previously assumed. As a general result that does not rely on any approximations we find that the DSEs directly exclude a class of IR fixed points where the IR strength arises directly from the gluon dynamics. Instead there are two other qualitatively different solutions for the fixed point behavior.

In the scaling solution the IR regime is strongly dominated by the ghost dynamics, as predicted e.g. in [11 16, 47]. The structure of the scaling fixed point established in these studies, however, has to be amended by additional kinematic singularities. The presence of these singularities is not only consistent with the uniform scaling rules but has both conceptual and quantitative impact on the structure of this solution. In a companion article [20] we present detailed analytic results for the 3-point vertices that give the complete kinematic dependence and show precisely the same kinematic divergences found here by pure power counting arguments. The knowledge about the leading dynamical contributions obtained here in combination with the analytic results for the 3-point functions should allow to give an improved value for the IR exponent $\kappa$ in an approximation that treats the 3 -point vertices dynamically. This should include the main dynamical contributions for a precise prediction of this pertinent parameter.

The non-perturbative analysis of kinematic divergences established here is even more important in the case of QCD. There non-perturbatively enhanced, strong kinematic divergences of the quark-gluon vertex in the corresponding scaling solution can provide a description of crucial aspects of QCD, like the linear rising potential between static color sources in the quenched theory [6] and its long-range screening in the dynamical case[7] as well as the U(1) anomaly [48].

Depending on the renormalization prescription there is another decoupling fixed point were the gluon acquires a mass and decouples [21 23]. Current lattice results for the propagators clearly favor this possibility [3]. Yet, we find that, aside from possible inherent problems of these analyses, cf. [36 38, in the decoupling scenario the gluonic vertices remain bare in the IR limit and thereby this fixed point shows no infrared enhancement at all. Correspondingly it does 


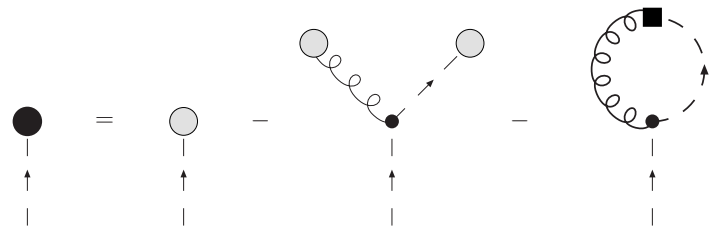

Figure 15: The generating ghost-DSE. Thin and thick lines and solid dots represent bare and proper propagators and vertices. Open dots represent explicit fields and the black square separating the two different lines denotes the corresponding off-diagonal component of the super-propagator.

not provide a description of the above vital aspects of QCD in terms of the Green functions of the fundamental local degrees of freedom alone. Despite its apparently simple form it would instead require a more complicated description of the QCD vacuum in terms of other degrees of freedom.

\section{Acknowledgments}

It is a pleasure to thank Christian Fischer, Felipe Llanes-Estrada, Axel Maas, Jan Pawlowski and Lorenz von Smekal for enlightening discussions. K.S. acknowledges support from the Austrian science fund (FWF) under contract M979N16, R.A. from the DFG under contract AL 279/5-2 and M.Q.H. is supported by the Doktoratskolleg "Hadrons in Vacuum, Nuclei and Stars" of the FWF under contract W1203-N08.

\section{Appendix A: Graphical derivation of Dyson-Schwinger equations}

The Dyson-Schwinger equations for the vertices given in the main text were derived via an algorithmic method presented in [26]. In this appendix we sketch this method to derive Dyson-Schwinger equations for general correlation functions from the corresponding equation for the 1-point function which we will in the following refer to as the "generating DSE". They involve except for one bare vertex only proper correlation functions and are given for the ghost and gluon in figs. 15 and 16, respectively. As shown in detail in [26] within a convenient superfield formalism all other DSEs can be computed from these equations via the replacement rules in fig. 17] where the double lines stand for superfields that include all elementary fields in the theory. As the final step all expressions have to be evaluated at their vacuum expectation value which corresponds graphically to replacing all super-propagators and vertices by the irreducible ones in all possible ways that involve only physical propagators and vertices in accordance with the symmetry of the action. This generally removes many graphs, so that when performing this procedure it is useful to take into account beforehand to what order the DSEs shall be computed to neglect any unphysical terms that would already vanish anyhow during the extension steps.

In the special case of non-Abelian gauge theory the super-multiplet containing gluon and (anti-)ghost fields is given by $\phi=(A, \bar{c}, c)$ and denoted by curly and dashed lines respectively. Using the general graphical replacement rules fig. 17 yields in a straightforward way the equations for the leading correlation functions figs. 1 to 6 . The proper symmetry factors arise simply from different ways of obtaining the same graph in the above replacements steps. The arising super-propagators in the 2-loop term of the generating gluon-DSE fig. 16 only become relevant for the equation for the ghost-gluon vertex and corresponding higher order correlation functions. For the derivation of other correlation functions they may be replaced by ordinary gluon propagators. It is important to note, however, that it is not possible to derive the equation for the gluonic vertices by additional derivatives of the propagator equation fig. 2 since one would miss additional loop contributions from the next to last graph in fig. 16 that guarantee the symmetry of the corresponding equations in a perturbative approximation. Finally we remark, that since there are no fundamental quark-ghost interactions in QCD the graphical expressions for the generating quark DSE and the equations for the leading correlation functions are identical to the corresponding ghost equations displayed in fig. 15 respectively figs. 1. 3 and 4 . 


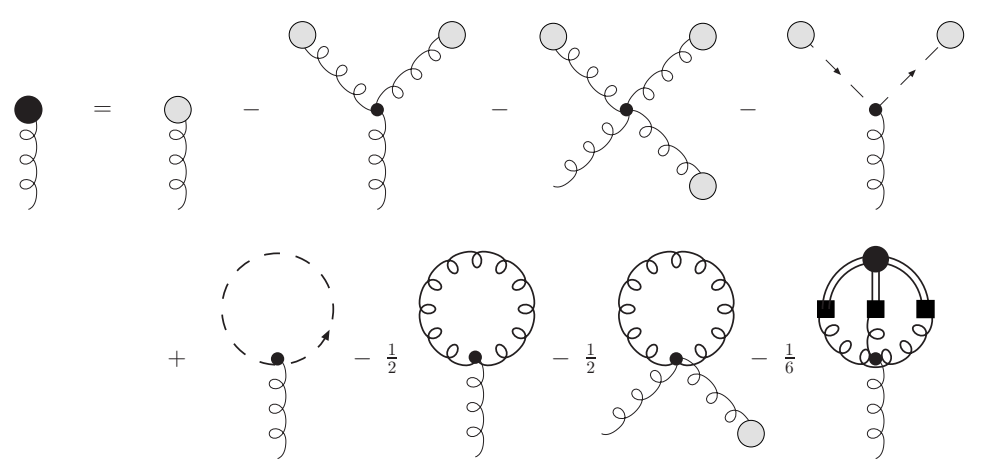

Figure 16: The generating gluon-DSE. The lhs in the generating equations represents the field derivative of the effective action.

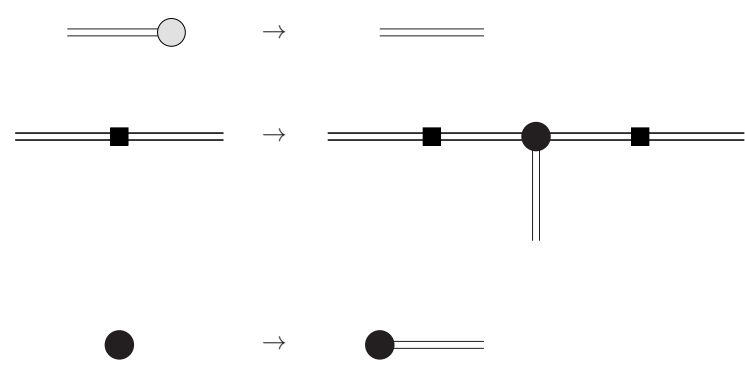

Figure 17: The replacement rules for the generation of DSEs for general correlation functions. One replacement step corresponds to a functional derivative that generates the DSE for the corresponding correlation function with one more external leg. In such a step one of the objects on the lhs is replaced by the corresponding expression on the rhs. Namely, an explicit field is simply removed. A general off-shell propagator is extended introducing a new proper 3-point vertex where each double line can stand for any field in the super-multiplet. A general proper vertex represented by the thick dot (which can already have any number of external legs) is simply extended via attaching another leg.

\section{Appendix B: Decomposition of the loop integrals}

\section{Massless integrals}

In this appendix we demonstrate explicitly how the loop integrals in the DSEs can be decomposed into different parts when both soft and hard external scales are present as the IR limit is taken. A general 1-loop graph is shown in fig. 18. The external momenta $q_{n}$ are assumed to be divided into two disjunct subsets $\left\{q_{n}\right\}=\left\{s_{i}\right\} \cup\left\{h_{j}\right\}$. The $s_{i}$ are soft and the $h_{j}$ hard, so that in the IR limit $\left|s_{i}\right| \ll\left|h_{j}\right| \forall i, j$. Similarly the linear combinations that can be formed out of these momenta and arise in the integral lines of a general loop graph,

$$
Q_{n}=\sum_{i_{n}} \zeta_{i_{n}} q_{i_{n}}
$$

with $\zeta_{i} \in\{-1,0,1\}$, can likewise be divided into corresponding subsets $\left\{Q_{n}\right\}=\left\{S_{i}\right\} \cup\left\{H_{j}\right\}$ with $\left|S_{i}\right| \ll\left|H_{j}\right| \forall i, j$. The integral $\mathcal{I}$ for a particular term in the general loop integral $\mathcal{G}$, eq. (6), arising from the expansion of the product of decomposed vertices eq. (2) reads then

$$
\mathcal{I}=\int \frac{d^{d} k}{(2 \pi)^{d}} \mathcal{K}_{\left\{l_{v}\right\}}\left(\left\{(k+Q)^{2}\right\},\left\{Q^{2}\right\}\right) \prod_{n}\left(k+Q_{n}\right)^{2\left(\delta_{n}-1\right)} \prod_{a} p_{l_{a}}^{2}\left(\left\{(k+Q)^{2}\right\},\left\{Q^{2}\right\}\right)^{\delta_{a}^{l_{a}}}
$$

where $n$ runs over the propagators and $a$ over the vertices in the graph. Here we skip the indices when denoting the corresponding sets arising as arguments of the functions $\mathcal{K}$ and $p$ defined in eqs. (6) and (2). The contributions to the 


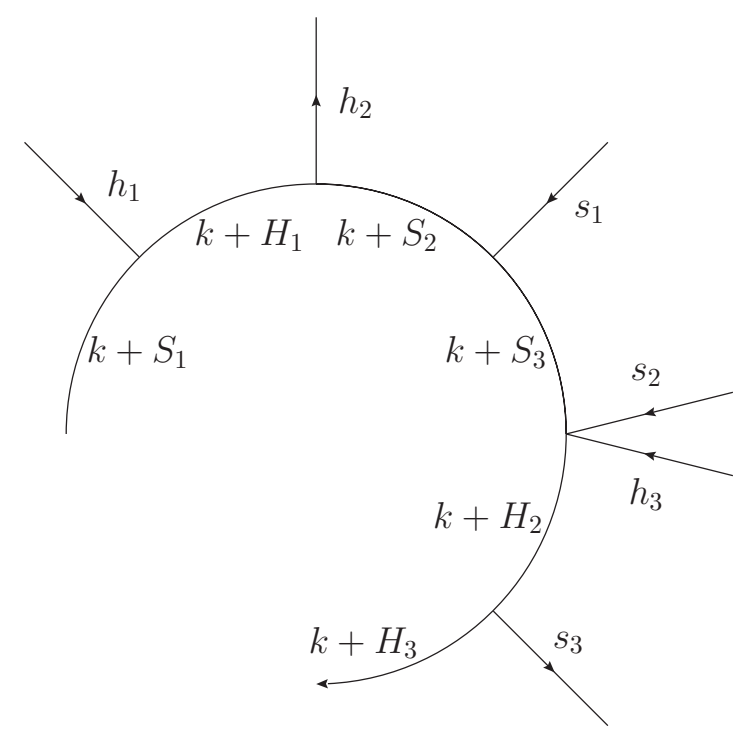

Figure 18: Exemplary momentum routing for a general $n$-point function. Here the arrows denote the momentum flow and the particular linear combinations arising in the diagram are given by $S_{1}=0, H_{1}=h_{1}, S_{2}=h_{1}-h_{2} \ll h_{1}, h_{2}, S_{3}=h_{1}-h_{2}+s_{1}$, $\cdots$.

vertex functions with different IR limits in the decomposition eq. 2) can be divided into a subset where the power law contains hard external momenta and another that does not

$$
\begin{gathered}
\mathcal{I}=\int \frac{d^{d} k}{(2 \pi)^{d}} \mathcal{K}_{\left\{l_{v}\right\}}\left(\left\{(k+S)^{2}\right\},\left\{S^{2}\right\},\left\{(k+H)^{2}\right\},\left\{H^{2}\right\}\right) \prod_{i}\left(k+S_{i}\right)^{2\left(\delta_{i}-1\right)} \prod_{j}\left(k+H_{j}\right)^{2\left(\delta_{j}-1\right)} \\
\cdot \prod_{a} p_{l_{a}}^{2}\left(\left\{(k+S)^{2}\right\}\right)^{\delta_{a}^{l_{a}}} \prod_{b} p_{l_{b}}^{2}\left(\left\{(k+S)^{2}\right\},\left\{(k+H)^{2}\right\}\right)^{\delta_{b}^{l_{b}}} .
\end{gathered}
$$

In the IR limit one can choose some intermediate scale $\Theta$, with $S \ll \Theta \ll H$ where $S \equiv \max \left(\left|S_{i}\right|\right)$ and $H \equiv \min \left(\left|H_{j}\right|\right)$, which can e.g. be chosen as $\Theta \equiv \sqrt{S H}$. Then the loop integral can be split into two parts

$$
\begin{aligned}
\mathcal{I} & =\left(\int_{0}^{\Theta} d k+\int_{\Theta}^{\infty} d k\right) k^{d-1} \int \frac{d \Omega_{d}}{(2 \pi)^{d}} \mathcal{K}_{\left\{l_{v}\right\}}(\cdots) \prod_{i}\left(k+S_{i}\right)^{2\left(\delta_{i}-1\right)} \prod_{j}\left(k+H_{j}\right)^{2\left(\delta_{j}-1\right)} \prod_{a} p_{l_{a}}^{2}(\cdots)^{\delta_{a}^{l_{a}}} \prod_{b} p_{l_{b}}^{2}(\cdots)^{\delta_{b}^{l_{b}}} \\
& \equiv \mathcal{I}_{<}+\mathcal{I}_{>}
\end{aligned}
$$

Consider first $\mathcal{I}_{<}$, where the factors involving the $H_{j} \gg \Theta$ can be expanded in $k, S_{i}$

$$
\begin{aligned}
\mathcal{I}_{<}=\mathcal{K}_{\left\{l_{v}\right\}}\left(\left\{H^{2}\right\}\right) \prod_{j} H_{j}^{2\left(\delta_{j}-1\right)} \prod_{b} p_{l_{b}}^{2}\left(\left\{H^{2}\right\}\right)^{\delta_{b}^{l_{b}}} \\
\quad \cdot \int_{0}^{\Theta} d k k^{d-1} \int \frac{d \Omega_{d}}{(2 \pi)^{d}} \prod_{i}\left(k+S_{i}\right)^{2\left(\delta_{i}-1\right)} \prod_{a} p_{l_{a}}^{2}\left(\left\{(k+S)^{2}\right\}\right)^{\delta_{a}^{l_{a}}}\left(1+\sum_{j} O\left(\frac{|k|}{\left|H_{j}\right|}\right)\right) .
\end{aligned}
$$

Here the analyticity of the $p_{i}$ as well as the partial analyticity of $\mathcal{K}$ has been used. Note that possible non-analyticities of $\mathcal{K}_{\left\{l_{v}\right\}}$ at hard momenta are no problem as far as the external momenta $q_{i}$ of the Green function themselves do not represent a singular configuration at hard external momenta. As discussed in the main text such a physical pole occurs in Euclidean space away from the real axis and directly at such a point the solution of the Dyson-Schwinger equations is not well defined anyhow and we can therefore simply exclude this special kinematic case here. The integral can now be extended over all scales. The leading part is 


$$
\mathcal{I}_{<}=\mathcal{K}_{\left\{l_{v}\right\}}\left(\left\{H^{2}\right\}\right) \prod_{j} H_{j}^{2\left(\delta_{j}-1\right)} \prod_{b} p_{l_{b}}^{2}\left(\left\{H^{2}\right\}\right)^{\delta_{b}^{l_{b}}} \int_{0}^{\infty} d k(\cdots)-\Delta \mathcal{I}_{<}+\tilde{O}\left(\frac{\Theta}{H}\right)
$$

where here and in the following the symbol $\tilde{O}$ means that subleading terms are of the given relative order and correspondingly suppressed compared to the leading terms that are given explicitly. In the correction term the other factors can be expanded

$$
\Delta \mathcal{I}_{<}=\mathcal{K}_{\left\{l_{v}\right\}}\left(\left\{H^{2}\right\}\right) \prod_{j} H_{j}^{2\left(\delta_{j}-1\right)} \prod_{b} p_{l_{b}}^{2}\left(\left\{H^{2}\right\}\right)^{\delta_{b}^{l_{b}}} \int_{\Theta}^{\infty} d k \int \frac{d \Omega_{d}}{(2 \pi)^{d}} k^{2\left(\sum_{i}\left(\delta_{i}-1\right)+\frac{d-1}{2}\right)} \prod_{a} p_{l_{a}}^{2}\left(k^{2}\right)^{\delta_{a}^{l_{a}}}\left(1+O\left(\frac{\left|S_{i}\right|}{|k|}\right)\right) .
$$

Analogously one can expand the upper part of the integral

$$
\begin{aligned}
\mathcal{I}_{>}= & \int_{\Theta}^{\infty} d k \int \frac{d \Omega_{d}}{(2 \pi)^{d}} \mathcal{K}_{\left\{l_{v}\right\}}\left(\left\{(k+H)^{2}\right\},\left\{H^{2}\right\}\right) k^{2\left(\sum_{i}\left(\delta_{i}-1\right)+\frac{d-1}{2}\right)} \prod_{j}\left(k+H_{j}\right)^{2\left(\delta_{j}-1\right)} \\
& \cdot \prod_{a} p_{l_{a}}^{2}\left(k^{2}\right)^{\delta_{a}^{l_{a}}} \prod_{b} p_{l_{b}}^{2}\left(k^{2},\left\{(k+H)^{2}\right\}\right)^{\delta_{b}^{l_{b}}}\left(1+\sum_{i} O\left(\frac{\left|S_{i}\right|}{|k|}\right)\right)=\int_{0}^{\infty} d k(\cdots)-\Delta \mathcal{I}_{>}+\tilde{O}\left(\frac{S}{\Theta}\right)
\end{aligned}
$$

and subsequently the correction term so that their sum gives

$$
\begin{aligned}
\Delta \mathcal{I} & =\Delta \mathcal{I}_{<}+\Delta \mathcal{I}_{>} \\
& =\mathcal{K}_{\left\{l_{v}\right\}}\left(\left\{H^{2}\right\}\right) \prod_{j} H_{j}^{2\left(\delta_{j}-1\right)} \prod_{b} p_{b}^{2}\left(\left\{H^{2}\right\}\right)^{\delta_{b}} \int_{0}^{\infty} d k \int \frac{d \Omega_{d}}{(2 \pi)^{d}} k^{2\left(\sum_{i}\left(\delta_{i}-1\right)+\frac{d-1}{2}\right)} \prod_{a} p_{l_{a}}^{2}\left(k^{2}\right)^{\delta_{a}^{l_{a}}}+\tilde{O}\left(\frac{S}{\Theta}, \frac{\Theta}{H}\right) .
\end{aligned}
$$

This integral involves no scale and thereby simply represents a, possibly infinite, constant multiplying its prefactor. This prefactor is in particular completely independent of soft scales and $\Delta \mathcal{I}$ does thereby not affect the IR power laws of the corresponding Green functions. In the case of primitively divergent Green functions considered in this work such a constant contribution is already present in the DSEs in the form of the tree level term. Moreover, for primitively divergent Green functions the integrals $\Delta \mathcal{I}$ can be explicitly UV divergent. Such a divergence cancels by construction precisely the additional UV divergence introduced in the additional scale dependent integral via eq. (B6). Correspondingly, this constant contribution could be formally absorbed into the multiplicative renormalization factor of the tree level term, which thereby has the form to cancel all divergences in both scale dependent integrals. We note that e.g. in dimensional renormalization the above scale independent integral vanishes by definition. Thereby, after proper renormalization the initial integral is split into two finite contributions that are in the IR limit $S \ll H$ independent of the scale $\Theta$

$$
\begin{aligned}
& \mathcal{I}=\mathcal{K}_{\left\{l_{v}\right\}}\left(\left\{H^{2}\right\}\right) \prod_{j} H_{j}^{2\left(\delta_{j}-1\right)} \prod_{b} p_{l_{b}}^{2}\left(\left\{H^{2}\right\}\right)^{\delta_{b}^{l_{b}}} \int \frac{d^{d} k}{(2 \pi)^{d}} \prod_{i}\left(k+S_{i}\right)^{2\left(\delta_{i}-1\right)} \prod_{a} p_{l_{a}}^{2}\left(\left\{(k+S)^{2}\right\}\right)^{\delta_{a}^{l_{a}}}(\mathrm{~B} 10) \\
& +\int \frac{d^{d} k}{(2 \pi)^{d}} \mathcal{K}_{\left\{l_{v}\right\}}\left(\left\{(k+H)^{2}\right\},\left\{H^{2}\right\}\right) \prod_{j}\left(k+H_{j}\right)^{2\left(\delta_{j}-1\right)} \prod_{b} p_{l_{b}}^{2}\left(k^{2},\left\{(k+H)^{2}\right\}\right)^{\delta_{b}^{l_{b}}} k^{2 \sum_{i}\left(\delta_{i}-1\right)} \prod_{a} p_{l_{a}}^{2}\left(k^{2}\right)^{\delta_{a}^{l_{a}}}+\tilde{O}\left(\frac{S}{H}\right) .
\end{aligned}
$$

The first integral depends now only on soft external momenta, whereas the second one depends only on hard momenta. These integrals are generally dominated by the poles of the integrand and have to scale with the respective external scales. Correspondingly once the contributions from scales of different order of magnitude are separated their scale dependence can be analyzed by mere power counting.

Yet, the hard integral can still be subject to soft singularities when differences of the hard momenta become soft. Then the formally absent soft momenta re-enter via the condition of momentum conservation. To see this, consider a 3-point integral with one soft and two hard external momenta. For better readability we skip in the following the vertex dressing functions and the kernel $\mathcal{K}$ but note that the derivation proceeds analogously with these complications. The decomposition eq. B10 reads then

$$
\mathcal{I}^{(3)}=h^{2(\alpha+\beta-2)} \int \frac{d^{d} k}{(2 \pi)^{d}}(k-s)^{2(\gamma-1)}+\int \frac{d^{d} k}{(2 \pi)^{d}}(k+h-s)^{2(\alpha-1)}(k+h)^{2(\beta-1)} k^{2(\gamma-1)}+\tilde{O}\left(\frac{|s|}{|h|}\right)
$$


The momentum combination $k+h-s$ in the hard part of the loop integral is again sensitive to the soft momentum $s$ in a small region around the loop momentum $k=-h$. To conveniently study this region it is useful to shift the integration variable via the substitution $k^{\prime}=k+h$ in order to divide the integral at some scale $\Theta$

$\mathcal{I}_{>}^{(3)}=\int \frac{d^{d} k^{\prime}}{(2 \pi)^{d}}\left(k^{\prime}-s\right)^{2(\alpha-1)} k^{\prime 2(\beta-1)}\left(k^{\prime}-h\right)^{2(\gamma-1)}=\left(\int_{0}^{\Theta} d k^{\prime}+\int_{\Theta}^{\infty} d k^{\prime}\right) \int \frac{d \Omega_{d}}{(2 \pi)^{d}}\left(k^{\prime}-s\right)^{2(\alpha-1)} k^{2\left(\beta+\frac{d-3}{2}\right)}\left(k^{\prime}-h\right)^{2(\gamma-1)}$.

In complete analogy to the general case above (using $s_{1}=s, s_{2}=0, h_{1}=-h$ and $\delta_{i=1}=\alpha, \delta_{i=2}=\beta$, $\delta_{j=1}=\gamma$ ) this integral can then be decomposed once more so that the full 3-point integral is effectively divided into three distinct integrals extending over all scales

$\mathcal{I}^{(3)}=h^{2(\alpha+\beta-2)} \int \frac{d^{d} k}{(2 \pi)^{d}}(k-s)^{2(\gamma-1)}+h^{2(\gamma-1)} \int \frac{d^{d} k}{(2 \pi)^{d}}(k-s)^{2(\alpha-1)} k^{2(\beta-1)}+\int \frac{d^{d} k}{(2 \pi)^{d}} k^{2(\alpha+\beta-2)}(k+h)^{2(\gamma-1)}+\tilde{O}\left(\frac{|s|}{|h|}\right)$.

The first two integrals are, up to $\tilde{O}(|s| /|h|)$ corrections, completely IR dominated whereas the third one is dominated by hard scales. This division is graphically displayed in fig. 10 in the main text by distinct loop graphs with colorcoded soft and hard propagators. The case of 2-loop contributions in the DSEs can be handled accordingly, with the complication that both loop integrals have to be split and the number of distinct integrals increases. In general the number of possible divisions depends on the $n$-point function, the number of loops and the numbers of soft and hard external momenta. After a full decomposition of the integrals there are - aside from purely hard integrals that yield a scale independent constant - only integrals left that depend on a given set of soft external momenta $\left\{s_{i}\right\}$. These momenta can be parameterized in the form $s_{i}=\rho_{i} s$ with a single scaling variable $s$ and fixed, bounded momentum ratios $\rho_{i} \equiv s_{i} / s,\left|\rho_{i}\right| \leq 1$ that could likewise be replaced by angular variables. In such a case the qualitative IR scaling of the corresponding integral is independent of the $\rho_{i}$ and can be determined by a pure power counting analysis. The prefactor of the IR scaling law however involves the ratios $\rho_{i}$ and requires an explicit solution of the integral [20. For the cases we study in this work, the integrals that need to be decomposed depend even on a single soft scale and therefore trivially scale as some power of it.

\section{Massive integrals}

In this subsection we extend the previous decomposition of loop integrals to the case when mass scales are involved. As already discussed in the main text, in the case that all propagators in an integral are massive one can in the IR limit $p \ll m$ simply expand the whole integral, e.g.

$$
\int d^{d} k \frac{1}{(k+p)^{2}+m^{2}} \frac{1}{k^{2}+m^{2}} \approx \int d^{d} k \frac{1}{\left(k^{2}+m^{2}\right)^{2}}+\tilde{O}\left(\frac{|p|}{m}\right)
$$

to convince oneself that the integral does not depend on the soft external scale to leading order. Even though there is no pole here the integral is dominated by modes of the order of the mass: the contribution from low modes is suppressed by phase space and the renormalized contribution from large modes is suppressed by the denominators. When there are both massive and massless propagators present the integral receives contributions from both soft and hard scales. To see this consider the massive 2-point integral of the form

$$
\mathcal{I}_{m} \equiv \int d^{d} k \frac{1}{(k+p)^{2 \alpha}} \frac{1}{k^{2}+m^{2}}
$$

In the limit $p \ll m$ one can again introduce an arbitrary intermediate scale $\Theta$ and divide the integrals 


$$
\begin{aligned}
\mathcal{I}_{m} & =\left(\int_{0}^{\Theta} d k k^{d-1} \int d \Omega+\int_{\Theta}^{\infty} d k k^{d-1} \int d \Omega\right) \frac{1}{(k+p)^{2 \alpha}} \frac{1}{k^{2}+m^{2}} \\
& =\frac{1}{m^{2}} \int_{0}^{\Theta} d k k^{d-1} \int d \Omega \frac{1}{(k+p)^{2 \alpha}}\left(1+O\left(\frac{|k|}{m}\right)\right)+\int_{\Theta}^{\infty} d k k^{d-1} \int d \Omega \frac{1}{k^{2 \alpha}} \frac{1}{k^{2}+m^{2}}\left(1+O\left(\frac{|p|}{|k|}\right)\right) \\
& =\frac{1}{m^{2}} \int_{0}^{\infty} d^{d} k \frac{1}{(k+p)^{2 \alpha}}+\int_{0}^{\infty} d^{d} k \frac{1}{k^{2 \alpha}} \frac{1}{k^{2}+m^{2}}-\frac{1}{m^{2}} \int_{0}^{\infty} d^{d} k \frac{1}{k^{2 \alpha}}+\tilde{O}\left(\frac{|p|}{m}\right)
\end{aligned}
$$

where the scale independent integral again cancels momentum independent divergences introduced in the decomposition. The first integral is dominated by soft scales and the second one by hard scales of the order of the mass and correspondingly the scale separation in the IR limit allows again the use of a power counting analysis. An analogous decomposition is possible for massive vertex integrals as well as for integrals that involve both masses and hard external momentum scales. In all these cases the presented decomposition results in a complete separation of the various external scales in different loop integrals and allows thereby the application of power counting methods. Finally we note that the presented method for decomposing massive integrals is likewise suitable for multi-loop corrections, like the 2-loop graphs arising in certain DSEs, via successive decompositions.

[1] R. Alkofer and L. von Smekal, Phys. Rept. 353 (2001) 281, hep-ph/0007355

[2] C. S. Fischer, J. Phys. G 32 (2006) R253 arXiv:hep-ph/0605173.

[3] P. O. Bowman et al., Phys. Rev. D 76 (2007) 094505 arXiv:hep-lat/0703022; I. L. Bogolubsky et al., Phys. Rev. D 77 (2008) 014504 [Erratum-ibid. D 77 (2008) 039902] [arXiv:0707.3611][hep-lat]]; A. Cucchieri, and T. Mendes, PoS LAT2007 (2007) 297 arXiv:0710.0412 [hep-lat]].

[4] T. Kugo and I. Ojima, Prog. Theor. Phys. Suppl. 66 (1979) 1; T. Kugo, arXiv:hep-th/9511033

[5] V. N. Gribov, Nucl. Phys. B 139 (1978) 1; D. Zwanziger, Nucl. Phys. B 364 (1991) 127.

[6] R. Alkofer, C. S. Fischer, F. J. Llanes-Estrada and K. Schwenzer, Annals Phys. 324 (2009) 106 [arXiv:0804.3042 [hep-ph]].

[7] K. Schwenzer, arXiv:0811.3608 [hep-ph].

[8] J. Braun, H. Gies and J. M. Pawlowski, Phys. Lett. B 684 (2010) 262 [arXiv:0708.2413 [hep-th]].

[9] R. Alkofer, C. S. Fischer and F. J. Llanes-Estrada, Mod. Phys. Lett. A 23 (2008) 1105 arXiv:hep-ph/0607293; R. Alkofer, C. S. Fischer, F. J. Llanes-Estrada and K. Schwenzer, PoS LAT2007 (2007) 286 [arXiv:0710.1154 [hep-ph]].

[10] C. D. Roberts and A. G. Williams, Prog. Part. Nucl. Phys. 33 (1994) 477 arXiv:hep-ph/9403224.

[11] L. von Smekal, R. Alkofer and A. Hauck, Phys. Rev. Lett. 79 (1997) 3591, hep-ph/9705242 Annals Phys. 267 (1998) 1 [Erratum-ibid. 269 (1998) 182], hep-ph/9707327, C. S. Fischer, R. Alkofer and H. Reinhardt, Phys. Rev. D 65 (2002) 094008, hep-ph/0202195, C. S. Fischer and R. Alkofer, Phys. Lett. B 536 (2002) 177, hep-ph/0202202

[12] D. Zwanziger, Phys. Rev. D 65 (2002) 094039 arXiv:hep-th/0109224.

[13] C. Lerche and L. von Smekal, Phys. Rev. D 65 (2002) 125006 arXiv:hep-ph/0202194.

[14] R. Alkofer, C. S. Fischer and F. J. Llanes-Estrada, Phys. Lett. B 611 (2005) 279 arXiv:hep-th/0412330].

[15] C. S. Fischer and J. M. Pawlowski, Phys. Rev. D 75 (2007) 025012 arXiv:hep-th/0609009; Phys. Rev. D 80 (2009) 025023 arXiv:0903.2193 [hep-th]].

[16] M. Q. Huber, R. Alkofer, C. S. Fischer and K. Schwenzer, Phys. Lett. B 659 (2008) 434, arXiv:0705.3809 [hep-ph].

[17] J. C. Taylor, Nucl. Phys. B 33 (1971) 436.

[18] Ph. Boucaud et al., JHEP 0703 (2007) 076 arXiv:hep-ph/0702092.

[19] S. Mandelstam, Phys. Rev. D 20 (1979) 3223.

[20] R. Alkofer, M. Q. Huber and K. Schwenzer, Eur. Phys. J. C 62 (2009) 761 arXiv:0812.4045 [hep-ph]].

[21] Ph. Boucaud, J. P. Leroy, A. L. Yaouanc, J. Micheli, O. Pene and J. Rodriguez-Quintero, JHEP 0806 (2008) 012 arXiv:0801.2721 [hep-ph]].

[22] A. C. Aguilar, D. Binosi and J. Papavassiliou, Phys. Rev. D 78 (2008) 025010 arXiv:0802.1870 [hep-ph]].

[23] C. S. Fischer, A. Maas and J. M. Pawlowski, Annals Phys. 324 (2009) 2408 [arXiv:0810.1987 [hep-ph]].

[24] J. M. Cornwall, Phys. Rev. D 26 (1982) 1453.

[25] D. Dudal, J. A. Gracey, S. P. Sorella, N. Vandersickel and H. Verschelde, Phys. Rev. D 78 (2008) 065047 arXiv:0806.4348 [hep-th]].

[26] R. Alkofer, M. Q. Huber and K. Schwenzer, Comput. Phys. Commun. 180 (2009) 965, arXiv:0808.2939 [hep-th].

[27] M. Q. Huber, K. Schwenzer and R. Alkofer, arXiv:0904.1873 [hep-th].

[28] C. Anastasiou, E. W. N. Glover and C. Oleari, Nucl. Phys. B 572 (2000) 307 hep-ph/9907494.

[29] E. E. Boos and A. I. Davydychev, Moscow Univ. Phys. Bull. 42N3 (1987) 6 [Vestn. Mosk. Univ. Fiz. Astron. 28N3 (1987) 8].

[30] W. Schleifenbaum, A. Maas, J. Wambach and R. Alkofer, Phys. Rev. D 72 (2005) 014017 arXiv:hep-ph/0411052.

[31] C. Kellermann and C. S. Fischer, Phys. Rev. D 78 (2008) 025015 [arXiv:0801.2697 [hep-ph]]. 
[32] J. Berges, Phys. Rev. D 70 (2004) 105010 arXiv:hep-ph/0401172.

[33] J. M. Pawlowski, private discussion.

[34] C. S. Fischer, A. Maas, J. M. Pawlowski and L. von Smekal, Annals Phys. 322 (2007) 2916 arXiv:hep-ph/0701050.

[35] A. Cucchieri and T. Mendes, Phys. Rev. Lett. 100 (2008) 241601 [arXiv:0712.3517 [hep-lat]].

[36] A. Sternbeck, E. M. Ilgenfritz, M. Mueller-Preussker and A. Schiller, Phys. Rev. D 72 (2005) 014507 arXiv:heplat/0506007.

[37] A. Maas, Phys. Rev. D 79 (2009) 014505 arXiv:0808.3047 [hep-lat]].

[38] A. Sternbeck and L. von Smekal, arXiv:0811.4300 [hep-lat].

[39] A. Maas, arXiv:0907.5185 [hep-lat].

[40] M. Q. Huber, R. Alkofer and S. P. Sorella, Phys. Rev. D 81 (2010) 065003 [arXiv:0910.5604 [hep-th]].

[41] D. Zwanziger, Nucl. Phys. B 323 (1989) 513.

[42] A. Maas, Phys. Rev. D 75 (2007) 116004 arXiv:0704.0722 [hep-lat]]; A. Cucchieri, T. Mendes and A. R. Taurines, Phys. Rev. D 67 (2003) 091502 arXiv:hep-lat/0302022.

[43] A. Cucchieri, A. Maas and T. Mendes, Phys. Rev. D 77 (2008) 094510 arXiv:0803.1798 [hep-lat]l, Phys. Rev. D 74 (2006) 014503 arXiv:hep-lat/0605011; A. Cucchieri, T. Mendes and A. Mihara, JHEP 0412 (2004) 012 arXiv:hep-lat/0408034.

[44] T. Schafer and K. Schwenzer, Phys. Rev. Lett. 97 (2006) 092301 arXiv:hep-ph/0512309, Phys. Rev. D 70 (2004) 054007 arXiv:hep-ph/0405053.

[45] R. Alkofer, P. Bicudo, S. R. Cotanch, C. S. Fischer and F. J. Llanes-Estrada, arXiv:nucl-th/0601032

[46] K. Schwenzer, et al., in preparation

[47] D. Zwanziger, Phys. Rev. D 69 (2004) 016002 arXiv:hep-ph/0303028.

[48] R. Alkofer, C. S. Fischer and R. Williams, Eur. Phys. J. A 38 (2008) 53, arXiv:0804.3478 [hep-ph]. 